\title{
EXTENSIONS OF ALGEBRAIC SYSTEMS
}

BY

\author{
AWAD A. ISKANDER
}

\begin{abstract}
There are several generalizations to universal algebras of the notion "The group $\mathfrak{A}$ is an extension of the group $\mathscr{V}$ by the group $(5)$. In this paper we study three such generalizations and the corresponding products of classes of algebraic systems. Various results are presented. One such theorem characterizes the weakly congruence regular varieties admitting extensions of a particular sort. Another result gives, under a weak congruence permutability condition, an equational basis for the variety obtained by applying one such product to two other varieties.
\end{abstract}

The product of group varieties $\mathscr{Q}$ and $\mathscr{V}$ is defined as the class of all groups that are Schreier extensions of $\mathscr{Q}$-groups by $\mathcal{T}$-groups [28, p. 38]. This class multiplication turns the set of all group varieties into a free monoid with 0 (B. H. Neumann, H. Neumann, and P. Neumann and independently Šmelkin [28, p. 58]). In [23], A. I. Mal'cev suggested the following definition of class multiplication for algebraic systems:

Definition 1 (MAL'CEV [23]). Suppose $\mathcal{K}$ is a class of algebraic systems and $\mathscr{Q}, \mathcal{T}$ are subclasses of $\mathscr{K} \cdot \mathscr{U} \cdot \mathscr{K} \mathfrak{V}$ is the class of all $\mathscr{K}$-systems $\mathfrak{A}$ such that a quotient $\mathfrak{U} / \boldsymbol{\theta} \in \mathcal{V}$ and every $\boldsymbol{\theta}$-block that is a $\mathfrak{K}$-system is also in $\mathscr{Q}$.

This class multiplication specializes to the well-known product of group varieties. Several interesting properties and applications of this class multiplication are considered in [23] (for an English translation, see [25, Chapter 32]). In [38] Žitomirskiı suggested an extension for single universal algebras that generalizes Schreier's [33] group extensions, Clifford's [4] and Redei's [30] semigroup extensions, and Everett's [9] ring extensions. To formulate his definition we need some preliminaries. For any subsystem $\mathfrak{B}$ of an algebraic system $\mathfrak{A}$, the set of all congruences on $\mathfrak{U}$ such that $B$ is a union of blocks is a principal ideal of the congruence lattice of $\mathfrak{A}$.

DEFINITION 2. If $\mathfrak{B}$ is a subsystem of an algebraic system $\mathfrak{A}, \boldsymbol{\theta}(\mathfrak{A}, \mathfrak{B})$ is the largest congruence on $\mathfrak{A}$ such that $B$ is a union of blocks.

Definition 3 (Ž́ItomirskiI [38]). Suppose $\mathfrak{A}, \mathfrak{B}$, $\mathfrak{C}$ are algebraic systems. $\mathfrak{A}$ is an extension of $\mathfrak{B}$ by $\mathfrak{C}$ if there is a subsystem $\mathfrak{B}^{\prime}$ of $\mathfrak{A}$ such that $\mathfrak{B}^{\prime} \cong \mathfrak{B}$ and $\mathfrak{U} / \boldsymbol{\theta}\left(\mathfrak{U}, \mathfrak{B}^{\prime}\right) \cong \mathfrak{E}$.

Received by the editors August 7, 1979 and, in revised form, February 2, 1983.

1980 Mathematics Subject Classification. Primary 08B05, 08B10, 08C15; Secondary 03C05, 03C60.

Key words and phrases. Classes of algebraic systems, universal algebras, expansions, extensions, weakly congruence permutable varieties, weakly congruence regular varieties, predicates, terms, identities, varieties with ideals, admitting extensions. 
The following is another generalization for the product of group varieties:

Definition 4. Suppose $\mathscr{K}$ is a class of algebraic systems and $\mathscr{U}, \mathcal{V}$ are subclasses of $\mathcal{K} . \mathscr{Q} *_{K} \mathcal{T}$ is the class of all $\mathcal{K}$-systems that are extensions of $\mathscr{Q}$-systems by T-systems.

The requirements of Definition 4 make some of our arguments a bit complicated. So we suggest the following:

Definition 5. Suppose $\mathfrak{A}, \mathfrak{B}, \mathfrak{C}$ are algebraic systems. $\mathfrak{A}$ is an expansion of $\mathfrak{B}$ by $\sqrt{5}$ if there are a subsystem $\mathfrak{B}^{\prime}$ of $\mathfrak{A}$ and a congruence $\theta$ on $\mathfrak{A}$ such that $\mathfrak{B}^{\prime} \cong \mathfrak{B}$, $\mathfrak{A} / \theta \cong \mathbb{E}$ and $B^{\prime}$ is a union of $\theta$-blocks. If $\mathscr{U}, \mathcal{V}$ are subclasses of a class $\mathcal{K}$ of algebraic systems, $\mathscr{Q} \circ_{\mathfrak{K}} \mathcal{T}$ is the class of all $\mathcal{K}_{-}$-systems that are expansions of Q-systems by $\mathcal{T}$-systems.

In the present paper we consider some properties of, and connections between $\cdot_{K}, *_{\mathscr{K}}$ and $\circ \mathscr{K}$. If $\mathcal{Q}, \mathcal{T}, \mathcal{K}$ are quasivarieties, then $\mathscr{U} \cdot \mathscr{K}$ is a quasivariety (Mal'cev [23]), and if $\mathcal{K}$ has also a constant term, then $\mathscr{Q} \circ \mathscr{T}$ is a quasivariety. If $\mathcal{V}$ is closed under the formation of homomorphic images, then $\mathscr{Q} \circ \mathscr{K}=$ $\mathcal{Q} *_{\mathscr{K}} \mathfrak{V}$. A unit is a singleton system on which all basic predicates are true; a unit term is a unary term all of whose values are units. A weakly congruence regular variety contains with any two of its members $\mathfrak{A}, \mathfrak{B}$ an extension of $\mathfrak{A}$ by $\mathfrak{B}$ iff it has a unit term. We show that if $\mathcal{Q}, \mathcal{T}$ are subvarieties of a weakly congruence permutable variety $\mathscr{K}$ (every member has a unit $e$ such that for any congruences $\theta, \Phi$ and any element $x, e \theta \Phi x$ iff $e \Phi \theta x$ ) of algebras, then $\mathcal{Q} \circ \mathcal{K}=\mathcal{U}_{\kappa} \mathscr{V}$ is closed under homomorphic images and $\mathcal{Q} \cdot \mathcal{K} \mathcal{V}$ is the largest subvariety of $\mathscr{U} \circ_{\mathcal{K}} \mathcal{T}$. Weak congruence permutability together with weak congruence regularity imply congruence Arguesianness and congruence $n$-permutability. Finally we determine the identities of $\mathscr{U} \cdot \mathcal{K}$ 作 subvarieties $\mathscr{Q}, \mathcal{V}$ of a weakly congruence permutable variety $\mathscr{K}$. This form of identities yields the corresponding identities for varieties of groups, loops, idempotent quasigroups, multioperator groups and rings.

Most of the characterization theorems of this paper are Mal'cev type conditions. An extensive theory of Mal'cev type conditions is available in Taylor [36]. Weakly congruence regular varieties are characterized by Grätzer [12]. Congruence regular varieties are characterized by Csákány [6], Grätzer [12] and Wille [37]. Varieties in which a block of every congruence is a subalgebra are characterized by Csákány [7]. Congruence Arguesianness is discussed in Jónsson [17, 18] and Schmidt [31, p. 59]. Congruence $n$-permutability is considered by Grätzer [12], Hagemann and Mitschke [13], Mitschke [27], Quakenbush [29], Schmidt [31, pp. 32, 53] and Wille [37]. The varieties of algebras with ideals of Fichtner [10] are precisely the varieties of algebras with constant terms that are weakly congruence regular and contain with any two members an extension of the first by the second. General references in universal algebra are Birkhoff [2, Chapter 6], Cohn [5], Grätzer [11], Henkin, Monk and Tarski [14, Chapter 0], and Mal'cev [24]; also Bell and Slomson [1] for models and ultraproducts. Unless otherwise stated, the notations are those of Grätzer [11]. We will use the same symbol for a term (polynomial)-symbol as well as its value in a given algebraic system. The word "system" will mean "algebraic system" in the sense of Mal'cev [24] or "structure" in the sense of Grätzer [11]. The word "algebra" 
will mean "universal algebra". A homomorphism $\alpha$ of a system $\mathfrak{A}$ into a system $\mathfrak{B}$ is a homomorphism of the algebra reducts such that whenever $P\left(a_{1}, \ldots, a_{n}\right)$ is true in $\mathfrak{A}$, for some predicate $P, P\left(a_{1} \alpha, \ldots, a_{n} \alpha\right)$ is true in $\mathscr{B}$; if $\alpha$ is onto, then $\mathscr{B}$ is a homomorphic image of $\mathfrak{A}$, under $\alpha$, if whenever $P\left(b_{1}, \ldots, b_{n}\right)$ is true in $\mathscr{B}$, $P\left(a_{1}, \ldots, a_{n}\right)$ is true in $\mathfrak{A}$ for some $a_{1}, \ldots, a_{n}$ such that $a_{i} \alpha=b_{i}, i=1, \ldots, n$. The phrase "a class of systems" will mean "a class of systems of the same type closed under isomorphisms".

The author is very grateful to the referee for an exceptionally thoughtful critique that improved the paper considerably.

1. In this section we consider some connections between class multiplications: $\cdot_{\mathcal{K}}, *_{\mathscr{K}}, \circ_{\mathscr{K}}$.

Lemma 1.1. If $\mathcal{U}, \mathcal{V}$ are subclasses of a class $\mathscr{K}$ of systems, then $\mathcal{U} *_{\mathscr{K}} \mathscr{V} \subseteq \mathcal{U} \circ \mathscr{K}$.

LEMmA 1.2. If $\mathfrak{U}, \mathfrak{B}, \mathfrak{C}$ are systems and $\mathfrak{A}$ is an expansion of $\mathfrak{B}$ by $\mathfrak{E}$, then $\mathfrak{A}$ is an extension of $\mathfrak{B}$ by a quotient of $\mathbb{E}$.

LEMMA 1.3. If $\mathscr{U}$ is a subclass of a class $\mathcal{K}$ of systems, then $\mathscr{U} \subseteq \mathscr{Q} \circ_{K} \mathscr{U}$.

Proof. If $\mathfrak{A} \in \mathscr{U}$, then $\mathfrak{A}$ is an expansion of $\mathfrak{A}$ by $\mathfrak{A}$, where $\theta=\Delta(\mathfrak{A})=$ the diagonal congruence on $\mathfrak{A}$.

LEMma 1.4. If $\mathscr{Q}, \mathcal{T}$ are subclasses of a class $\mathcal{K}$ of systems and $\mathcal{T}$ is closed under

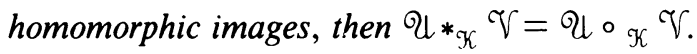

This follows from Lemmas 1.1 and 1.2.

Lemma 1.5. Suppose $\mathcal{K}$ is a class of systems closed under subsystems and $\mathscr{Q}, \mathfrak{V}$ are subclasses of $\mathcal{K}$. If every $\mathfrak{T}$-system contains a singleton subsystem, then $\mathscr{U} \cdot \mathcal{K} \subseteq$ थ०\% $\mathcal{T}$.

Proof. Let $\mathfrak{A} \in \mathcal{U} \cdot \mathscr{K} \mathfrak{V}$. Then there is a congruence $\boldsymbol{\theta}$ on $\mathfrak{A}$ such that $\mathfrak{A} / \boldsymbol{\theta} \in \mathfrak{V}$ and every $\theta$-block that is a $\mathcal{K}_{-}$-system is in $\mathscr{U}$. As $\mathfrak{A} / \boldsymbol{\theta} \in \mathcal{V}$, it contains a singleton subsystem. Hence, a $\theta$-block is a subsystem of $\mathfrak{A} \in \mathscr{K}$. Thus, for some $a \in A$, $[a] \theta \in \mathscr{K}$, since $\mathscr{K}$ is closed under subsystems. Hence $[a] \theta \in \mathcal{Q}$. Thus $\mathfrak{U}$ is an expansion of $[a] \theta \in \mathcal{Q}$ by $\mathfrak{A} / \boldsymbol{\theta} \in \mathcal{V}$.

It is not true, in general, that $\mathscr{U} \cdot \mathcal{K}-\mathcal{Q} \circ \mathscr{K} T=\varnothing$. Indeed, if $\mathcal{K}$ is the variety of all groupoids, and $\mathcal{U}$ is the subvariety of all semigroups and $\mathfrak{A}$ is the free groupoid on one free generator $a$, then $\mathfrak{U} \in \mathscr{U} \cdot \varkappa \mathscr{U}-\mathscr{U} \circ_{\kappa} \mathscr{U}$. Define $x \theta y$ iff the numbers of entries of $a$ in $x$ and $y$ are equal. $\theta$ is a congruence on $\mathfrak{A}$ and $\mathfrak{A} / \theta \in \mathscr{U}$. Since for no $x \in A, x \theta(x)(x)$, no $\theta$-block is a subgroupoid of $\mathfrak{A}$. Thus $\mathfrak{A} \in \mathscr{U} \cdot \mathscr{K}$ U. However, no subgroupoid of $\mathfrak{A}$ is associative. Indeed, for no triple of natural numbers $(x, y, z), x @(y @ z)=(x @ y) @ z$, where@ is the operation on the natural numbers defined by $x @ y=2^{x} 3^{y}$. Hence $\mathfrak{A} \notin$ U० $x$ U.

For the reverse inclusion, we can prove

Lemma 1.6. Suppose $\mathcal{K}$ is a class of systems and $\mathscr{U}, \mathcal{T}$ are subclasses of $\mathcal{K}$. If $\mathcal{U}$ is closed under subsystems, every थ-system contains a singleton subsystem and every $\mathcal{T}$-system contains at most one singleton subsystem, then $\mathcal{Q} \circ_{\mathcal{K}} \mathcal{V} \subseteq \mathcal{U} \cdot \mathcal{K}$. 
Proof. Suppose $\mathfrak{A} \in \mathfrak{K}, \boldsymbol{\theta}$ is a congruence on $\mathfrak{A}$, and $\mathfrak{B}$ is a subsystem of $\mathfrak{A}$ such that $B$ is a union of $\theta$-blocks, $\mathfrak{B} \in \mathcal{Q}$ and $\mathfrak{A} / \theta \in \mathscr{V}$. Let $\{e\}$ be a singleton subsystem of $\mathfrak{B}$. Then $[e] \theta$ is a subsystem of $\mathfrak{B}$; i.e., $[e] \theta \in \mathcal{A} \mathscr{U} \subseteq \mathcal{U}$. If $[f] \theta$ is a subsystem of $\mathfrak{A}$, then $\{[f] \theta\}$ is a singleton subsystem of $\mathfrak{A} / \theta \in \mathcal{T}$. Hence $[e] \theta=$ $[f] \boldsymbol{\theta}$. Hence $\mathfrak{A} \in \mathcal{Q} \cdot \mathscr{K} \mathfrak{T}$.

Combining Lemmas 1.5 and 1.6 we obtain

THEOREM 1.7. Suppose $\mathcal{Q}, \mathcal{T}$ are subclasses of a class $\mathscr{K}$ of systems. If $\mathcal{K}$ and $\mathscr{U}$ are

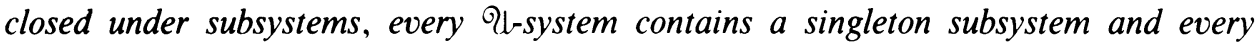
V-system contains exactly one singleton subsystem, then $\mathcal{U} \cdot{ }_{\hbar} \mathcal{T}=\mathcal{U} \circ_{\kappa} \mathcal{T}$.

Definition 1.1 (MAL'CEV [24, 25]). A system of type $\tau$ is a unit if it is a singleton system on which all $\tau$-predicates are true. An element $a$ of a system $\mathfrak{A}$ is a unit if it is the universe of a subsystem of $\mathfrak{A}$ that is a unit.

For algebras units and singleton algebras coincide. For algebras units are sometimes called idempotents.

Mal'cev observed [23] that $\%$ preserves class inclusion. Similar results are true for $*_{\mathscr{K}}$ and $\circ_{\overparen{K}}$. We summarize these results in the following theorem:

THEOREM 1.8. Suppose $\mathfrak{K}$ is a class of systems and $\mathscr{U}_{1}, \mathscr{U}_{2}, \mathscr{T}_{1}, \mathscr{T}_{2}$ are subclasses of $\mathcal{K}$. Let $\mathcal{E}$ be the class of all units of $\mathcal{K}$ and let $\mathcal{E}_{0}$ be the class of all singleton systems of $\mathcal{K}, \square=\cdot_{K}, *_{\mathcal{K}}$ or $\circ_{\mathcal{K}}$. Then:

$\mathcal{Q}_{1} \square \mathcal{V}_{1} \subseteq \mathcal{Q}_{2} \square \mathcal{V}_{2}$ if $\mathcal{U}_{1} \subseteq \mathcal{Q}_{2}$ and $\mathcal{V}_{1} \subseteq \mathcal{V}_{2}$;

$\mathcal{U}_{1} \square \mathcal{E} \subseteq \mathcal{U}_{1}$ and $\mathfrak{U}_{1} \square \mathcal{E}_{0} \subseteq \mathcal{U}_{1}$;

$\mathcal{U}_{1} \square \mathcal{E}=\mathcal{U}_{1}$ iff every $\mathcal{U}_{1}$-system has a unit quotient in $\mathcal{K}$;

$\mathcal{U}_{1} \square \mathcal{E}_{0}=\mathcal{U}_{1}$ iff every $\mathcal{U}_{1}$-system has a singleton quotient in $\mathcal{K}$;

$\mathcal{E} \circ \mathscr{V}_{1} \supseteq \mathcal{V}_{1}$ iff every $\mathscr{V}_{1}$-system contains a unit subsystem in $\mathscr{K}$;

$\mathcal{E}_{0} \circ \mathcal{T}_{1} \supseteq \mathcal{T}_{1}$ iff every $\mathcal{V}_{1}$-system contains a singleton subsystem in $\mathcal{K}$.

Definition 1.2 (MAL'CEV [23, 24, 25]). Suppose $\mathfrak{V}$ is a class of systems and $\mathfrak{A}$ is a system of the same type as $\mathcal{T} . \theta$ is called a $\mathcal{T}$-congruence on $\mathfrak{A}$ if $\mathfrak{U} / \theta \in \mathcal{V}$ and if $\alpha$ is a homomorphism of $\mathfrak{A}$ into $\mathfrak{B} \in \mathcal{V}$, then there is a homomorphism $\beta$ of $\mathfrak{A} / \theta$ into $\mathfrak{B}$ such that $\alpha$ is the composition of the natural homomorphism of $\mathfrak{A}$ into $\mathfrak{A} / \boldsymbol{\theta}$ and $\beta$.

The following lemma was proved by Mal'cev [23, Corollary 4; 25, p. 425] for ' $\gamma$. The similar result for ${ }^{\circ} \mathscr{K}$ can be easily proved.

Lemma 1.9. Suppose $\mathfrak{K}$ is a class of systems, $\mathfrak{A} \in \mathfrak{K}$ and $\mathscr{U}, \mathcal{T}$ are subclasses of $\mathscr{K}$. If $\theta$ is a $\mathfrak{V}$-congruence on $\mathfrak{A}$, then $\mathfrak{A} \in \mathcal{Q} \cdot{ }_{\mathfrak{h}} \mathfrak{T}$ iff every $\theta$-block that is a $\mathcal{K}_{\text {-system is }}$ in $\mathcal{Q l}$.

A variety of algebras is called regularly defined $[21,35]$ if its type does not include nullary operations and if it has a basis of the form $p\left(x_{0}, \ldots, x_{n-1}\right)=q\left(x_{0}, \ldots, x_{n-1}\right)$ where $p$ and $q$ are terms depending on precisely the same variables.

THEOREM 1.10. Suppose $\mathfrak{K}$ is a regularly defined variety of algebras and $\mathfrak{T}$ is a regularly defined subvariety of $\mathcal{K}$. If there is a simple algebra in $\mathscr{K}-\mathcal{T}$, then $\mathcal{V} *_{K} \mathcal{V}=\mathcal{V} \circ \ldots \mathcal{T} \neq \mathcal{V} \cdot_{K} \mathcal{T}$. 
Proof. Since $\mathcal{H} \mathscr{T} \subseteq \mathcal{V}, \mathcal{V} *_{\mathcal{K}} \mathcal{V}=\mathscr{V} \circ_{\mathscr{K}} \mathcal{V}$ by Lemma 1.4. We suppose the reader is familiar with $\S 2$ of [21], and we use the notations and results from there.

Suppose $\mathfrak{A}$ is a simple algebra in $\mathscr{K}-\mathcal{T}$ and $\mathfrak{U}^{\prime}$ is a one element extension of $\mathfrak{A}$, i.e., $A^{\prime}=A \cup\{e\}$, where $e \notin A$ and $f\left(x_{1}, \ldots, x_{n}\right)=e$ if some $x_{i}=e$ and $f\left(a_{1}, \ldots, a_{n}\right)$ is defined as in $\mathfrak{U}$ if $a_{1}, \ldots, a_{n} \in A$, for every operation $f$. Then by [21], $\mathfrak{A}^{\prime} \in \mathscr{K}$. If $\theta$ is the equivalence relation with classes $A$ and $\{e\}$, then $\theta$ is a congruence relation on $\mathfrak{U}^{\prime}$ and $\mathfrak{U}^{\prime} / \theta \cong 2 \tau$ where $\tau$ is the type of $\mathscr{K}$. Thus $\mathfrak{U}^{\prime} / \theta \in \mathcal{V}$. $[e] \theta=\{e\} \in \mathcal{T}$. So $\mathfrak{H}^{\prime} \in \mathcal{T} \circ_{\mathscr{K}} \mathcal{T}$. For any $a \in A,[a] \theta=A \notin \mathcal{T}$.

Suppose $\Phi$ is a congruence on $\mathfrak{A}^{\prime}$ and $\theta \not \Phi$. Then the restriction of $\Phi$ to $\mathfrak{A}$ is the diagonal congruence on $\mathfrak{A}$. So $\mathfrak{A}^{\prime} / \Phi \notin \mathcal{V}$. So $\theta$ is a $\mathcal{T}$-congruence on $\mathfrak{A}^{\prime}$. By Lemma 1.9, we have $\mathfrak{A}^{\prime} \notin \mathfrak{V} \cdot \mathscr{K} \mathfrak{T}$.

For a specific example, one may take $\mathscr{K}=$ the variety of all semigroups and $\widetilde{V}=$ the variety of all commutative semigroups, since any nonabelian simple group is a noncommutative simple semigroup.

The ideas of the above proof can be modified and extended to provide other examples of algebras in $\mathcal{V} \circ_{\mathcal{K}} \mathcal{V}-\mathcal{V} \cdot \mathscr{K}$. The latter applies even in some cases where $\mathcal{K}$ and $\mathscr{V}$ are not regularly defined varieties. Even though the class of all lattices is not a regularly defined variety, we have a similar result.

THEOREM 1.11. Suppose $\mathfrak{A}$ is a subdirectly irreducible lattice and $\mathfrak{V}$ is a nontrivial lattice variety not containing $\mathfrak{U}$. If $((\mathcal{H} S\{\mathfrak{U}\})-\mathscr{G}\{\mathfrak{U}\}) \subseteq \mathcal{V}$, then $\mathcal{V} *_{\mathfrak{L}} \mathfrak{V}=\mathfrak{V} \circ_{\mathfrak{L}} \mathfrak{V} \neq$ $\tau \cdot{ }_{e} \mathcal{T}$.

Proof. By Lemma 1.4, $*_{\mathfrak{e}}=o_{\mathfrak{e}}$.

Let us consider the case when $\mathfrak{A}$ is simple. The lattice $\mathbb{E}$ obtained by adjoining to $\mathfrak{U}$ a new zero, which we denote by $0^{\prime}$, satisfies $\mathbb{S} \in \mathcal{V} \circ_{\mathfrak{L}} \mathfrak{V}$, $\mathbb{E} \notin \mathcal{V} \cdot \mathfrak{L} \mathcal{T}$. Indeed, the equivalence relation $\theta$ with classes $A$ and $\left\{0^{\prime}\right\}$ is a congruence on $\Subset$. $\Subset / \theta$ is a two element chain and belongs to $\mathscr{V}$. $\left[0^{\prime}\right] \theta=\left\{0^{\prime}\right\} \in \mathcal{V}$. So $\mathbb{E} \in \mathcal{V} \circ{ }_{\mathcal{L}} \mathcal{V}$. However, $\theta$ is the $\mathcal{T}$-congruence on $\mathcal{E}$ and $[a] \theta=A$ for every $a \in A$. Since $\mathfrak{A} \in \mathcal{L}$ and $\mathfrak{A} \notin \mathcal{V}$, we have $\mathbb{E} \notin \mathcal{V} \cdot \mathcal{Q} \mathcal{V}$ by Lemma 1.9 .

Suppose now that $\mathfrak{A}$ is not simple. Then $\mathfrak{A}$ contains an edge $e \ll e^{\prime}$; i.e., $e^{\prime}$ covers $e$ and $\theta\left(e, e^{\prime}\right)=\Psi$ is the least nontrivial congruence on $\mathfrak{A}$. Moreover, since $\mathfrak{A}$ is not simple, there is some $a \notin[e] \Psi$. Let $\Subset$ be the lattice obtained from $A \cup A_{1}$ where $\mathfrak{A}_{1}$ is a disjoint isomorphic copy of $\mathfrak{A}$ and $x \leqslant y$ in $\mathbb{E}$ iff $x \leqslant y$ in $\mathfrak{A}$, or $x \leqslant y$ in $\mathfrak{A}_{1}$, or $x \in A$ and $y \in A_{1}$ and $x \leqslant e$, or $x \in A_{1}$ and $y \in A$ and $e^{\prime} \leqslant y$. Let $\theta=\theta\left(e, e^{\prime}\right)$. Clearly $\mathfrak{E} / \theta \cong \mathfrak{A} / \Psi \in \mathcal{V}$. Also $[a] \theta=[a] \Psi$, but $[a] \Psi \in \mathfrak{V}$ by hypothesis. So (5) $\in \mathcal{T} \circ_{\mathrm{e}} \mathcal{T}$.

Moreover, $\theta$ is the least congruence on $\mathbb{E}$ such that $\mathbb{E} / \theta \in \mathcal{T}$. Indeed, suppose $\Phi$ is a congruence and $\theta \nsubseteq \Phi$. Then $\left(e, e^{\prime}\right) \notin \Phi$ and the restriction of $\Phi$ to $A$ is the equality relation. Hence $\mathfrak{\mho} / \Phi$ has a sublattice isomorphic to $\mathfrak{A} \notin \mathcal{V}$ and so $\mathfrak{\mho} / \Phi \notin \mathcal{T}$. Since $[e] \theta \supseteq A_{1} \in \mathcal{L}-\mathcal{V}$, by Lemma 1.9 , we have $\mathbb{E} \notin \mathcal{V} \cdot \mathfrak{L} \mathcal{V}$.

Actually, if $\mathscr{U}$ is a locally finite lattice variety and $\mathfrak{U} \in \mathscr{U}$ is finite and subdirectly irreducible, $\mathfrak{A}$ contains more than two elements, then the class $\mathscr{T}$ of all $\mathscr{W}$-lattices $\mathfrak{B}$ such that $\mathfrak{A} \notin H S \mathfrak{B}$ is a variety satisfying the conditions of Theorem 1.11 ( $\mathfrak{A}$ is a 
splitting lattice for $\mathcal{Q}$, see [16]). If $\mathscr{V}$ is one of the following lattice varieties: $\mathfrak{R}=$ all modular lattices, $\mathscr{D}=$ all distributive lattices, or a variety generated by a finite nontrivial lattice, then $\mathcal{V} \circ_{\mathfrak{e}} \mathfrak{V} \neq \mathcal{V} \cdot \mathfrak{L}$.

Splitting properties of lattices are considered in $[20,26]$. Relations between lattice varieties and subdirectly irreducibles are discussed in $[16,19]$.

2. In this section we consider preservation of $\circ_{\mathscr{K}}$ under the operators of formation of subsystems $(\mathcal{S})$, homomorphic images $(\mathcal{H})$, Cartesian products $(\mathscr{P})$ and ultraproducts.

LEMma 2.1. Suppose $\mathscr{K}$ is a class of systems with a constant term and $\mathcal{U}, \mathcal{V}$ are subclasses of $\mathcal{K}$. If $\mathscr{K}, \mathcal{Q}, \mathcal{T}$ are closed under subsystems, then $\mathscr{U} \circ \mathscr{K}$ is closed under subsystems. If also $\mathcal{H} \mathfrak{\mathcal { V }} \subseteq \mathcal{T}$, then $\mathscr{U} *_{\mathfrak{K}} \mathcal{T}$ is closed under subsystems.

Proof. Let $\mathfrak{A} \in \mathscr{U} \circ \mathscr{K} \mathfrak{T}$ and let $\mathfrak{B}$ be a subsystem of $\mathfrak{A}$. Thus $\mathfrak{B} \in \mathscr{K}$ and there are a congruence $\theta$ on $\mathfrak{A}$ and a subsystem $\mathbb{C}$ of $\mathfrak{A}$ such that $C$ is a union of $\theta$-blocks, $\mathfrak{F} \in \mathcal{U}$ and $\mathfrak{A} / \boldsymbol{\theta} \in \mathcal{V}$. $\mathfrak{S} \cap \mathfrak{B} \in \mathcal{Q}, C \cap B(\neq \varnothing)$ is a union of $\theta^{\prime}$-blocks where $\theta^{\prime}$ is the restriction of $\theta$ to $\mathfrak{B} . \mathfrak{B} / \boldsymbol{\theta}^{\prime}$ is isomorphic to a subsystem of $\mathfrak{A} / \boldsymbol{\theta}$; i.e., $\mathfrak{B} / \boldsymbol{\theta}^{\prime} \in \mathcal{S} \mathcal{V}$ $\subseteq \mathcal{V}$. Thus $\mathfrak{B}$ is an expansion of $\mathbb{C} \cap \mathfrak{B} \in \mathcal{U}$ by $\mathfrak{B} / \boldsymbol{\theta}^{\prime} \in \mathcal{T}$.

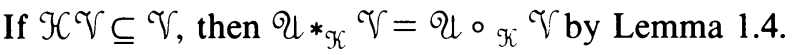

LEMma 2.2. Suppose $\mathcal{K}$ is a class of systems and $\mathcal{U}, \mathcal{T}$ are subclasses of $\mathcal{K}$. If $\mathscr{K}$, $\mathcal{Q}$, $\mathcal{V}$ are closed under Cartesian products, then $\mathscr{U} \circ \mathscr{K} \mathcal{V}$ is closed under Cartesian products. If also $\mathcal{H} \mathfrak{V} \subseteq \mathcal{T}$, then $\mathscr{Q} *_{\mathcal{K}} \mathfrak{V}$ is closed under Cartesian products.

Proof. Let $\mathfrak{A}_{i} \in \mathscr{U} \circ \mathscr{K} \mathfrak{V}, i \in I$. There are congruences $\theta_{i}$ on $\mathfrak{A}_{i}$ and subsystems $\mathfrak{B}_{i}$ of $\mathfrak{A}_{i}$ such that $\mathfrak{B}_{i}$ is in $\mathcal{Q}, \mathfrak{B}_{i}$ is a union of $\theta_{i}$-blocks and $\mathfrak{A}_{i} / \theta_{i}$ is in $\mathfrak{T}$, for each $i \in I . \theta=\Pi\left\{\theta_{i} \mid i \in I\right\}$ is a congruence on

$$
\mathfrak{U}=\Pi\left\{\mathfrak{A}_{i} \mid i \in I\right\} \in \mathfrak{K}, \quad \mathfrak{U} / \theta \cong \Pi\left\{\mathfrak{A}_{i} / \theta_{i} \mid i \in I\right\} \in \mathcal{P} \mathscr{V} \subseteq \mathcal{V} .
$$

Also, $\mathfrak{B}=\Pi\left\{\mathfrak{B}_{i} \mid i \in I\right\}$ is a subsystem of $\mathfrak{A}, \mathfrak{B} \in \mathscr{P} \mathscr{U} \subseteq \mathscr{U}, B$ is a union of $\theta$-blocks. So $\mathfrak{A} \in \mathcal{U} \circ_{\mathscr{K}} \mathfrak{V}$.

LEMma 2.3. Suppose $\mathscr{K}$ is a class of systems and $\mathcal{U}, \mathcal{T}$ are subclasses of $\mathcal{K}$. If $\mathscr{K}$, $\mathcal{Q}$, $\mathcal{V}$ are closed under ultraproducts, then $\mathscr{Q} \circ \mathcal{K}$ is closed under ultraproducts. If $\mathcal{H} \mathfrak{V} \subseteq \mathcal{V}$, then $\mathscr{U} *_{\mathscr{K}} \mathfrak{V}$ is closed under ultraproducts.

Proof. Let $\mathfrak{A}_{i} \in \mathscr{U} \circ \mathscr{K} \mathfrak{T}, i \in I$ and let $\mathfrak{F}=\langle I ; F\rangle$ be an ultrafilter on $I$. Then $\mathfrak{U}=\Pi\left\{\mathfrak{A}_{i} \mid i \in I\right\} / \mathscr{F} \in \mathscr{K}$. There are subsystems $\mathfrak{B}_{i}$ of $\mathfrak{A}_{i}$, congruences $\theta_{i}$ on $\mathfrak{A}_{i}$ such that $\mathfrak{B}_{i} \in \mathcal{Q}, \mathfrak{A}_{i} / \theta_{i} \in \mathcal{V}$ and $B_{i}$ is a union of $\theta_{i}$-blocks for each $i \in I$. Expand the language by adding a binary relation symbol $\theta$ and a unary relation symbol $B$. For each $i$, interpret $\theta$ as $\theta_{i}$ and $B$ as $B_{i} . \theta$ being a congruence, $B$ being a subsystem and $B$ being a union of $\theta$-blocks are first order properties true in each expanded $\mathfrak{A}_{i}$ which we denote by $\mathfrak{A}_{i}^{\prime}$. $\Pi\left\{B_{i} \mid i \in I\right\} / \mathfrak{F}$ is isomorphic to the interpretation of $B$ in $\mathfrak{A}^{\prime}=\Pi\left\{\mathfrak{U}_{i}^{\prime} \mid i \in I\right\} / \mathfrak{F}, \boldsymbol{\theta}=\Pi\left\{\boldsymbol{\theta}_{i} \mid i \in I\right\} / \mathfrak{F}$ is a congruence on $\mathfrak{A} . \mathfrak{B}$ is a subsystem of $\mathfrak{A}$ and $B$ is a union of $\theta$-blocks.

$$
\mathfrak{U} / \boldsymbol{\theta} \cong \Pi\left\{\mathfrak{U}_{i} / \theta_{i} \mid i \in I\right\} / \mathfrak{F} \in \mathcal{V} .
$$

Thus $\mathfrak{A} \in \mathscr{Q} \circ \mathscr{K}$. 
Mal'cev showed that if $\mathscr{K}, \mathscr{Q}, \mathcal{V}$ are closed under subsystems (Cartesian products), then $\mathscr{U} \cdot \mathscr{K} \mathscr{T}$ is closed under subsystems (Cartesian products), without restrictions on the type of $\mathcal{K}$. Mal'cev also showed that if the type of $\mathscr{K}$ includes only a finite number of operation symbols, then $\mathscr{U} \cdot \mathscr{K} \mathscr{V}$ is closed under ultraproducts whenever $\mathcal{K}, \mathcal{Q}, \mathcal{V}$ are closed under ultraproducts.

THEOREM 2.4. Suppose $\mathfrak{K}$ is a class of systems with a constant term and $\mathcal{U}, \mathfrak{T}$ are subclasses of $\mathcal{K}$. If $\mathcal{K}, \mathcal{U}, \mathfrak{V}$ are defined by universal sentences, then $\mathscr{U} \circ \mathcal{K}$ T is defined by universal sentences.

Proof. A class is defined by universal sentences iff it is closed under subsystems and ultraproducts (cf. [1, 5, 11, 24]). Theorem 2.4 follows from Lemmas 2.1 and 2.3.

THEOREM 2.5. Suppose $\mathfrak{K}$ is a quasivariety of systems with a constant term and $\mathscr{Q}, \mathfrak{V}$ are subquasivarieties of $\mathcal{K}$. Then $\mathscr{U}_{\mathcal{K}} \mathfrak{V}$ is a quasivariety.

Proof. A quasivariety is a class defined by a set of quasi-equations; i.e., by a set of sentences in prenex normal form:

$$
\left(\forall x_{0} \cdots x_{n}\right)\left(\left(P_{1}\left(t_{1}, \ldots, t_{m}\right) \& \cdots \& P_{k}\left(t_{1}, \ldots, t_{m}\right)\right) \rightarrow P\left(t_{1}, \ldots, t_{m}\right)\right)
$$

where $P, P_{1}, \ldots, P_{k}$ are either predicates or $=$ and $t_{1}, \ldots, t_{m}$ are terms in $x_{0}, \ldots, x_{n}$. A class is a quasivariety iff it is closed under subsystems and Cartesian products and ultraproducts and contains a unit $[\mathbf{1}, \mathbf{5}, \mathbf{1 1}, \mathbf{2 4}]$. Theorem 2.5 follows from Lemmas 2.1-2.3.

If $\mathcal{L}$ is the class of all lattices, $\mathscr{Q}$ is the class of all one element lattices and $\mathscr{Q}$ is the class of all distributive lattices, then $\mathscr{Q}_{\mathcal{R}} \mathcal{Q}$ is not closed under sublattices and so is not a quasivariety. Even in varieties with nullary operations, $\mathscr{Q} \circ \mathscr{K} \widetilde{T}$ is not in general a variety in case $\mathcal{K}, \mathcal{Q}, \mathcal{V}$ are varieties. Indeed, $\mathcal{Q} \circ{ }_{\Re} \mathscr{Q}$ is not a variety, where $\mathfrak{N}=$ the variety of all monoids and $\mathscr{Q}=$ the variety of all commutative monoids.

3. In this section we consider classes admitting extensions and classes that are weakly congruence regular.

Definition 3.1. A class $\mathscr{K}$ of systems is said to admit extensions if for any $\mathfrak{A}, \mathfrak{B}$ in $\mathscr{K}$, there is $\mathbb{E} \in \mathcal{K}$, and $\mathbb{E}$ is an extension of $\mathfrak{A}$ by $\mathfrak{B}$.

Žitomirskii [38] showed that in the class $\mathscr{K}(\tau)$ of all algebras of type $\tau$, there is an extension of $\mathfrak{A}$ by $\mathfrak{B}$ iff there is a homomorphism $\alpha$ of $\mathfrak{A}$ into $\mathfrak{B}$ such that $\theta(\mathfrak{B}, \mathfrak{A} \alpha)=\Delta(\mathfrak{B})$. The proof of sufficiency depends on the fact that $\mathscr{K}(\tau)$ does not satisfy any nontrivial identities.

LeMma 3.1. Suppose $\mathcal{K}$ is a class of systems containing a unit and is closed under finite Cartesian products. Then $\mathcal{K}$ admits extensions iff every system $\mathfrak{A}$ of $\mathscr{K}$ contains a unit $e(\mathfrak{A})$ such that $\theta(\mathfrak{A}, e(\mathfrak{A}))=\Delta(\mathfrak{A})$.

Proof. Let $\mathscr{K}$ admit extensions and $\mathfrak{A} \in \mathcal{K}$. Then there is $\mathbb{E} \in \mathcal{K}$, $\mathbb{C}$ is an extension of $\mathbb{E}$ by $\mathfrak{A}$ where $\mathbb{E}$ is a unit of $\mathscr{K}$. Thus $\mathbb{E}$ contains a substem isomorphic

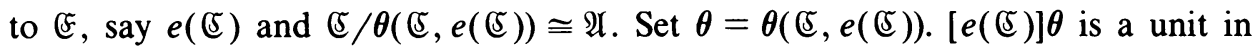
$\mathbb{E} / \theta$. Hence $\mathfrak{A}$ contains a unit $e=e(\mathfrak{A})$. Let $\Phi$ be a congruence on $\mathfrak{A}$ such that 
$[e] \Phi=\{e\}$. Hence there is a congruence $\Phi^{\prime}$ on $\mathfrak{S}, \Phi^{\prime} \geqslant \theta$ and $[e(\mathfrak{S})] \Phi^{\prime}=e(\mathfrak{S})$. Hence $\Phi^{\prime}=\boldsymbol{\theta}$ and $\Phi=\Delta(\mathfrak{A})$; i.e., $\boldsymbol{\theta}(\mathfrak{A}, e(\mathfrak{A}))=\Delta(\mathfrak{A})$.

Conversely, let $\mathfrak{A}, \mathfrak{B} \in \mathfrak{K}$ and $e(\mathfrak{A} \times \mathfrak{B})=(e, f)$ with $\theta(\mathfrak{A} \times \mathfrak{B}, \mathrm{e}(\mathfrak{A} \times \mathfrak{B}))=$ $\Delta(\mathfrak{U} \times \mathfrak{B})$. Then $\theta(\mathfrak{A},\{e\})=\Delta(\mathfrak{A})$ and $\theta(\mathfrak{B},\{f\})=\Delta(\mathfrak{B})$. Indeed, let $\Phi=\Delta(\mathfrak{A})$ $\times \theta$ where $\theta$ is a congruence on $\mathscr{B}$ such that $[f] \theta=\{f\}$. Hence $[(e, f)] \Phi=\{(e, f)\}$. Thus $\Phi=\Delta(\mathfrak{A} \times \mathfrak{B})$, and so $\theta=\Delta(\mathfrak{B}) . A \times\{f\}$ is a subsystem of $\mathfrak{A} \times \mathfrak{B}$ and $\mathfrak{A} \times\{f\}$ is a $\theta$-block where $(a, b) \theta\left(a^{\prime}, b^{\prime}\right)$ iff $b=b^{\prime} . \theta$ is a congruence on $\mathfrak{A} \times \mathfrak{B}$, $(\mathfrak{A} \times \mathfrak{B}) / \theta \cong \mathfrak{B}$ and $\theta=\theta(\mathfrak{A} \times \mathfrak{B}, \mathfrak{A} \times\{f\})$. Indeed, let $\Phi$ be a congruence on $\mathfrak{A} \times \mathfrak{B}$ such that $A \times\{f\}$ is a $\Phi$-block and $\Phi \geqslant \theta$. Set $b \Psi b^{\prime}$ iff there are $a, a^{\prime} \in A$ such that $(a, b) \Phi\left(a^{\prime}, b^{\prime}\right) . \Psi$ is a congruence on $\mathfrak{B}$. [ $\left.f\right] \Psi=\{f\}$. Thus $\Psi=\Delta(\mathfrak{B})$; i.e., $\Phi=\boldsymbol{\theta}$. Hence $\mathfrak{A} \times \mathfrak{B}$ is an extension of $\mathfrak{A}$ by $\mathfrak{B}$.

COROllaRy 3.2. If $\mathcal{T}$ is a quasivariety of systems, in particular if $\mathcal{V}$ is a variety, then $\mathcal{V}$ admits extensions iff every $\mathcal{W}$-system $\mathfrak{A}$ contains a unit $e(\mathfrak{H})$ such that $\theta(\mathfrak{A}, e(\mathfrak{A}))=$ $\Delta(\mathfrak{A})$.

This follows from Lemma 3.1 since quasivarieties contain units and are closed under Cartesian products.

Definition 3.2 (GrätZer [12]). A variety $\mathcal{T}$ of systems is called weakly congruence regular if for every system $\mathfrak{A} \in \mathcal{T}$ there is $0 \in A$ such that for any pair of congruences $\theta, \Phi$ if $[0] \theta=[0] \Phi$, then $\theta=\Phi$.

In [12] Grätzer proved that weakly congruence regular varieties of algebras are determined by a Mal'cev type condition. He showed that for such varieties there is a unary term $0\left(x_{0}\right)$ such that for any algebra $\mathfrak{A}, a \in A$ and any congruences $\theta, \Phi$ on $\mathfrak{A},[0(a)] \boldsymbol{\theta}=[0(a)] \Phi$ implies $\boldsymbol{\theta}=\Phi$. On the other hand Csákány [7] showed that varieties of algebras in which a block of every congruence is a subalgebra are precisely the varieties of algebras with a unit term.

PROPOSITION 3.3. Suppose $\mathcal{K}$ is a weakly congruence regular variety of systems. If $\mathfrak{U} \in \mathcal{K}$ and $e \in A$ is a unit, then for any pair of congruences $\theta, \Phi$ on $\mathfrak{A}, \boldsymbol{\theta}=\Phi$ iff $[e] \boldsymbol{\theta}=[e] \Phi$.

This follows from Grätzer's result because in this case $0(e)=e$.

THEOREM 3.4. For a variety $\mathcal{T}$ of systems, the following conditions are equivalent:

(i) $\mathcal{T}$ is weakly congruence regular and there is a unit term $e\left(x_{0}\right)$ on $\Upsilon$.

(ii) $\mathcal{T}$ is weakly congruence regular and admits extensions.

(iii) $\mathcal{T}$ is weakly congruence regular and a block of every congruence is a subsystem in which every basic predicate is satisfiable.

Proof. (i) $\leftrightarrow$ (ii) is immediate from Corollary 3.2 and Proposition 3.3. Also (ii) $\rightarrow$ (iii) is immediate. If $\mathcal{V}$ satisfies (iii), then a block of the diagonal congruence on the free $\mathcal{V}$-system of rank 1 is a subsystem in which every basic predicate is satisfiable; i.e., an element of the free $\mathcal{T}$-system on one generator is a unit. Thus (iii) $\rightarrow$ (i). 
THEOREM 3.5. For a variety $\mathcal{T}$ of systems, the following conditions are equivalent

(i) $\mathcal{T}$ is weakly congruence regular and admits extensions.

(ii) There are 3-ary terms $s_{i}\left(x_{0}, x_{1}, x_{2}\right), 1 \leqslant i \leqslant k$, such that $s_{1}\left(x_{0}, x_{0}, x_{0}\right)$ is a unit on $\mathcal{W}$ and for any $a, b, c \in \mathfrak{H} \in \mathfrak{T}, b=c$ iff $s_{i}(a, b, c)=s_{1}(a, a, a), 1 \leqslant i \leqslant k$.

(iii) There are 4-ary terms $p_{i j}\left(x_{0}, x_{1}, x_{2}, x_{3}\right), 0 \leqslant j \leqslant m(i), 1 \leqslant i \leqslant k$, 3-ary terms $t_{i}\left(x_{0}, x_{1}, x_{2}\right), 1 \leqslant i \leqslant k$, such that $t_{1}\left(x_{0}, x_{0}, x_{0}\right)$ is a unit,

$$
t_{i}\left(x_{0}, x_{1}, x_{1}\right)=t_{1}\left(x_{0}, x_{0}, x_{0}\right), \quad 1 \leqslant i \leqslant k,
$$

and

$$
\begin{gathered}
x_{1}=p_{10}\left(e, x_{1}, x_{2}, e\right), \quad x_{2}=p_{k m(k)}\left(e, x_{1}, x_{2}, t_{k}\left(e, x_{1}, x_{2}\right)\right), \\
p_{i j}\left(e, x_{1}, x_{2}, t_{i}\left(e, x_{1}, x_{2}\right)\right)=p_{i(j+1)}\left(e, x_{1}, x_{2}, t_{i}\left(e, x_{1}, x_{2}\right)\right) \quad \text { if } j \text { is even, } \\
p_{i j}\left(e, x_{1}, x_{2}, e\right)=p_{i(j+1)}\left(e, x_{1}, x_{2}, e\right) \quad \text { if } j \text { is odd }, \quad 0 \leqslant j<m(i), 1 \leqslant i \leqslant k, \\
p_{i m(i)}\left(e, x_{1}, x_{2}, t_{i}\left(e, x_{1}, x_{2}\right)\right)=p_{(i+1) 0}\left(e, x_{1}, x_{2}, e\right), \quad 1 \leqslant i<k,
\end{gathered}
$$

are identities in $\mathfrak{V}$ where $e=t_{1}\left(x_{0}, x_{0}, x_{0}\right)$.

Proof. Suppose (iii) is true. Set

$$
s_{i}\left(x_{0}, x_{1}, x_{2}\right)=t_{i}\left(t_{1}\left(x_{0}, x_{0}, x_{0}\right), x_{1}, x_{2}\right), \quad 1 \leqslant i \leqslant k .
$$

$s_{1}, \ldots, s_{k}$ satisfy (ii). Let $\mathfrak{V}$ satisfy (ii) and $\mathfrak{A} \in \mathcal{V}, a \in A$. Then $e=s_{1}(a, a, a)$ is a unit. Let $\boldsymbol{\theta}, \boldsymbol{\Phi}$ be congruences on $\mathfrak{A}$ such that $[e] \boldsymbol{\theta}=[e] \Phi$. $\mathfrak{A} / \boldsymbol{\theta} \in \mathcal{V}$. Hence $b \boldsymbol{\theta} c$ iff for all $1 \leqslant i \leqslant k$,

$$
s_{i}([e] \theta,[b] \theta,[c] \theta)=s_{1}(e[\theta],[e] \theta,[e] \theta) ;
$$

i.e., iff $s_{i}(e, b, c) \theta s_{1}(e, e, e)$. But $s_{1}(e, e, e)=e$. Thus $b \theta c$ iff $s_{i}(e, b, c) \in[e] \theta$, $1 \leqslant i \leqslant k$. Similarly $b \Phi c$ iff $s_{i}(e, b, c) \in[e] \Phi, 1 \leqslant i \leqslant k$. Since $[e] \theta=[e] \Phi, \theta=\Phi$. Thus $\mathcal{T}$ is weakly congruence regular and since it has a unit term $s_{1}\left(x_{0}, x_{0}, x_{0}\right)$, (i) follows from Theorem 3.4. It remains to prove that (i) $\rightarrow$ (iii).

Suppose that (i) is true and $\mathfrak{F}$ is the free $\mathcal{T}$-system freely generated by $x_{0}, x_{1}, x_{2}, \ldots$ By Corollary 3.2 and Proposition 3.3, there is a unit $f=f\left(x_{0}, \ldots, x_{n-1}\right) \in F$ such that for any pair of congruences $\theta, \Phi$ on $\mathfrak{F}, \theta=\Phi$ iff $[f] \theta=[f] \Phi$. Thus every congruence on $\mathfrak{F}$ is determined by its $f$-block, and so

$$
\theta\left(x_{n}, x_{n+1}\right)=\bigvee\left\{\theta(f, u) \mid u \in[f] \theta\left(x_{n}, x_{n+1}\right)\right\} .
$$

By the compactness of $\theta\left(x_{n}, x_{n+1}\right)$, there is a finite number of terms $u_{1}, \ldots, u_{k}$ such that $\left(f, u_{i}\right) \in \theta\left(x_{n}, x_{n+1}\right), 1 \leqslant i \leqslant k$ and

$$
\theta\left(x_{n}, x_{n+1}\right)=\bigvee\left\{\theta\left(f, u_{i}\right) \mid 1 \leqslant i \leqslant k\right\} .
$$

Thus $u_{i}=u_{i}\left(x_{0}, \ldots, x_{n-1}, x_{n}, x_{n+1}, \ldots, x_{m}\right)$ and $f=u_{i}\left(x_{0}, \ldots, x_{n-1}, x_{n}, x_{n}, \ldots, x_{m}\right)$ for all $1 \leqslant i \leqslant k$. There are $z_{1}, \ldots, z_{k+1} \in F$ such that $x_{n}=z_{1}, x_{n+1}=z_{k+1}$ and $\left(z_{i}, z_{i+1}\right) \in \theta\left(f, u_{i}\right), 1 \leqslant i \leqslant k$, at the expense of a repetition and a reordering of the $u_{i}$. Hence there are algebraic functions $q_{i j}(z)$ such that

$$
z_{i}=q_{i 0}(f), \quad z_{i+1}=q_{i m(i)}\left(u_{i}\right),
$$




$$
\begin{aligned}
& q_{i j}\left(u_{i}\right)=q_{i(j+1)}\left(u_{i}\right) \text { if } j \text { is even, } \\
& q_{i j}(f)=q_{i(j+1)}(f) \text { if } j \text { is odd, } \quad 0 \leqslant j<m(i), 1 \leqslant i \leqslant k \text {. }
\end{aligned}
$$

Since $z_{i+1}=q_{i m(i)}\left(u_{i}\right)$ and $z_{i+1}=q_{(i+1) 0}(f)$, then $q_{i m(i)}\left(u_{i}\right)=q_{(i+1) 0}(f)$ for all $1 \leqslant i \leqslant k$. (This follows from the general techniques of characterizing principal congruences due to A. I. Mal'cev [22] and its modifications in B. Jónsson [19] and G. Grätzer [12].)

Now, an algebraic function in one variable on $\mathfrak{f}$ can be identified by a term. Thus there are terms $r_{i j}\left(x_{0}, \ldots, x_{n-1}, x_{n}, x_{n+1}, \ldots, x_{s}, x_{s+1}\right)$ with $s \geqslant m$ such that

$$
\begin{gathered}
x_{0}=r_{10}\left(x_{0}, \ldots, x_{s}, f\right), \quad x_{n+1}=r_{k m(k)}\left(x_{0}, \ldots, x_{s}, u_{k}\left(x_{0}, \ldots, x_{m}\right)\right), \\
r_{i j}\left(x_{0}, \ldots, x_{s}, u_{i}\left(x_{0}, \ldots, x_{m}\right)\right)=r_{i(j+1)}\left(x_{0}, \ldots, x_{s}, u_{i}\left(x_{0}, \ldots, x_{m}\right)\right) \quad \text { if } j \text { is even, } \\
r_{i j}\left(x_{0}, \ldots, x_{s}, f\right)=r_{i(j+1)}\left(x_{0}, \ldots, x_{s}, f\right) \quad \text { if } j \text { is odd }, \quad 0 \leqslant j<m(i), 1 \leqslant i \leqslant k,
\end{gathered}
$$

and

$$
r_{i m(i)}\left(x_{0}, \ldots, x_{s}, u_{i}\left(x_{0}, \ldots, x_{m}\right)\right)=r_{(i+1) 0}\left(x_{0}, \ldots, x_{s}, f\right), \quad 1 \leqslant i<k .
$$

Let $\alpha$ be the endomorphism of $\mathfrak{F}$ defined by $x_{i} \alpha=e\left(x_{0}\right)=f\left(x_{0}, \ldots, x_{0}\right)$ if $i \neq n$, $i \neq n+1, i \neq s+1$ and $x_{n} \alpha=x_{1}, x_{n+1} \alpha=x_{2}, x_{s+1} \alpha=x_{3}$. The conditions of (iii) are satisfied when $t_{i}\left(x_{0}, x_{1}, x_{2}\right)=u_{i} \alpha, p_{i j}\left(x_{0}, x_{1}, x_{2}, x_{3}\right)=r_{i j} \alpha$, since $e\left(x_{0}\right)$ is a unit.

Special cases of weakly congruence permutable varieties admitting extensions are the varieties with ideals of K. Fichtner [10] and J. Slominski [34]. Such varieties are varieties with a unique "equationally defined" constant; they include, of course, multioperator groups, loops and rings. They are also varieties of algebras with unique units. The following lemma establishes conditions for such varieties:

LEMMA 3.6. For a variety $\mathfrak{V}$ of systems, the following conditions are equivalent:

(i) Every system of $\mathcal{V}$ contains precisely one unit.

(ii) There are unary terms $c\left(x_{0}\right)$ and $e\left(x_{0}\right)$ such that $c\left(x_{0}\right)=c\left(x_{1}\right)$ and $e\left(x_{0}\right)$ is a unit.

(iii) There is a unary term $e\left(x_{0}\right)$ such that $e\left(x_{0}\right)=e\left(x_{1}\right)$ and $e\left(x_{0}\right)$ is a unit.

Proof. It is clear that (iii) $\rightarrow$ (ii). If $\mathfrak{V}$ satisfies (ii) and $a \in \mathfrak{A} \in \mathfrak{V}$, then $e(a)$ is a unit. If $f \in A$ is a unit, then $f=c(f)=c(e(a))=e(a)$. Thus $\mathfrak{A}$ contains precisely one unit. Let $\mathfrak{V}$ satisfy (i) and let $\mathfrak{F}$ be the free $\mathfrak{V}$-system of rank 2 generated by $x_{0}, x_{1}$. Then $\mathfrak{F}$ has precisely one unit: $u\left(x_{0}, x_{1}\right)$. Thus $e\left(x_{0}\right)=u\left(x_{0}, x_{0}\right)$ and $e\left(x_{1}\right)$ are units. Hence $e\left(x_{0}\right)=e\left(x_{1}\right)$.

Thus if $\mathscr{V}$ is a variety of systems with constant terms and unit terms, then for every $\mathcal{V}$-system $\mathfrak{A}$, there is $0 \in A$ that is the value of all unit terms and constant terms.

Theorem 3.7. Suppose $\mathcal{T}$ is a variety of systems with a constant term. The following conditions on $\mathcal{T}$ are equivalent:

(i) $\mathcal{T}$ is weakly congruence regular and admits extensions.

(ii) There are binary terms $u_{i}\left(x_{0}, x_{1}\right), 1 \leqslant i \leqslant k$, such that $u_{1}\left(x_{0}, x_{0}\right)$ is a unit and for any system $\mathfrak{A} \in \mathcal{T}$, and any $a, b \in A, a=b$ iff $u_{i}(a, b)=u_{1}(a, a), 1 \leqslant i \leqslant k$. 
(iii) There are binary terms $v_{i}\left(x_{0}, x_{1}\right), 1 \leqslant i \leqslant k$, and ternary terms $q_{i j}\left(x_{0}, x_{1}, x_{2}\right)$, $0 \leqslant j \leqslant m(i), 1 \leqslant i \leqslant k$, such that $v_{1}\left(x_{0}, x_{0}\right)$ is a unit,

$$
v_{i}\left(x_{0}, x_{0}\right)=v_{1}\left(x_{0}, x_{0}\right), \quad 1 \leqslant i \leqslant k,
$$

and

$$
\begin{gathered}
x_{0}=q_{10}\left(x_{0}, x_{1}, 0\right), \quad x_{1}=q_{k m(k)}\left(x_{0}, x_{1}, v_{k}\left(x_{0}, x_{1}\right)\right), \\
q_{i j}\left(x_{0}, x_{1}, v_{i}\left(x_{0}, x_{1}\right)\right)=q_{i(j+1)}\left(x_{0}, x_{1}, v_{i}\left(x_{0}, x_{1}\right)\right) \quad \text { if } j \text { is even, } \\
q_{i j}\left(x_{0}, x_{1}, 0\right)=q_{i(j+1)}\left(x_{0}, x_{1}, 0\right) \quad \text { if } j \text { is odd, } \quad 0 \leqslant j<m(i), 1 \leqslant i \leqslant k,
\end{gathered}
$$

and

$$
q_{i m(i)}\left(x_{0}, x_{1}, v_{i}\left(x_{0}, x_{1}\right)\right)=q_{(i+1) 0}\left(x_{0}, x_{1}, 0\right), \quad 1 \leqslant i<k,
$$

where $0=v_{1}\left(x_{0}, x_{0}\right)$.

(iv) $\mathcal{T}$ is weakly congruence regular and has a unit term.

(v) $\mathcal{T}$ has a unit term that is also a constant term 0 and every congruence is determined by its 0-block (i.e., $\mathcal{T}$ is a variety with ideals [10].)

The equivalence of (ii), (v) and a variant of (iii) was established by Fichtner [10] for varieties of algebras with a nullary operation 0 that is also a unit.

Proof. By Lemma 3.6, a variety $\mathcal{V}$ with constant terms and unit terms is such that every $\widetilde{T}$-system contains a unique unit 0 ; it is also its unique constant. Theorem 3.7 follows from Theorem 3.5 and Lemma 3.6 where in all terms we replace $x_{0}$ by $0, x_{1}$ by $x_{0}, x_{2}$ by $x_{1}$ and $x_{3}$ by $x_{2}$.

The following theorem establishes some connections between varieties with unit terms [7], varieties with constant unit terms, congruence regular varieties $[6,12,37]$, varieties with ideals [10], idempotent varieties; i.e., every element of every system is a unit and Mal'cev's class multiplication [23].

THEOREM 3.8. Suppose $\mathcal{T}$ is a variety of systems with a unit term. The following conditions on $\mathcal{T}$ are equivalent:

(i) For some unit term e $\left(x_{0}\right)$ in $\mathscr{V}, \mathcal{T}$ satisfies

$$
e\left(f\left(x_{0}, \ldots, x_{n-1}\right)\right)=e\left(f\left(e\left(x_{0}\right), \ldots, e\left(x_{n-1}\right)\right)\right)
$$

for every operation $f$ of the type of $\mathcal{T}$.

(ii) For all operations $f$ and all unit terms $e$ of the type of $\mathcal{V}, \mathcal{V}$ satisfies (1).

(iii) $\mathfrak{V}=\mathcal{U}_{0} \cdot \mathcal{V} \mathcal{U}_{1}$ for some subvariety $\mathcal{U}_{0}$ of $\mathfrak{V}$ with a constant term and some idempotent subvariety $\mathscr{U}_{1}$ of $\mathscr{T}$.

(iv) $\mathfrak{V}=\mathcal{V}_{0} \cdot \mathcal{V} \mathcal{V}_{1}$ where $\mathcal{V}_{0}, \mathcal{V}_{1}$ are the subvarieties of $\mathscr{V}$ defined by $e\left(x_{0}\right)=e\left(x_{1}\right)$ and $x_{0}=e\left(x_{0}\right)$, respectively, for some unit term $e\left(x_{0}\right)$ in $\mathcal{T}$.

Proof. By Lemma 3.6, varieties with unit terms and constant terms are varieties where every system has exactly one unit, which is also its unique constant. $\mathcal{T}_{0}$ satisfies $e\left(x_{0}\right)=e\left(x_{1}\right)$; i.e., it is a variety with a constant term. $\mathcal{T}_{1}$ satisfies $x_{0}=e\left(x_{0}\right)$; i.e., it is idempotent. Thus (iv) $\rightarrow$ (iii). Let (iii) be true. Thus $\mathcal{U}_{1}$ satisfies $x_{0}=e\left(x_{0}\right)$ for any unit term $e\left(x_{0}\right)$. Also $\mathcal{Q}_{0}$ satisfies $e\left(x_{0}\right)=e\left(x_{1}\right)$ for any unit term $e$ by Lemma 3.6. Let $\mathfrak{A} \in \mathcal{V}$ and let $f$ be an $n$-ary operation of the type of $\mathcal{T}$. 
There is a congruence $\theta$ on $\mathfrak{A}$ such that $\mathfrak{A} / \theta \in \mathcal{Q l}$, and every $\theta$-block that is a subsystem of $\mathfrak{A}$ belongs to $\mathscr{Q}_{0}$. As every element in $\mathfrak{A} / \boldsymbol{\theta}$ is a unit, every $\theta$-block is a subsystem of $\mathfrak{A}$. Thus $[a] \theta \in \mathcal{Q l}_{0}$ for every $a \in A$. Hence $a \theta e(a)$ for all $a \in A$. Thus, for any $a_{1}, \ldots, a_{n} \in A, a_{i} \in \theta e\left(a_{i}\right), 1 \leqslant i \leqslant n$. So $f\left(a_{1}, \ldots, a_{n}\right) \theta f\left(e\left(a_{1}\right), \ldots, e\left(a_{n}\right)\right)$. Since $e\left(x_{0}\right)=e\left(x_{1}\right)$ is true in each $\theta$-block, $e\left(f\left(a_{1}, \ldots, a_{n}\right)\right)=e\left(f\left(e\left(a_{1}\right), \ldots, e\left(a_{n}\right)\right)\right)$. Thus (iii) $\rightarrow$ (ii). It is immediate that (ii) $\rightarrow$ (i). Suppose (i) is true. The relation $x \theta y$ iff $e(x)=e(y)$ is a congruence on $\mathfrak{A} \in \Upsilon^{\top}$ by (i). $e(e(x))=e(x)$ since $e$ is a unit term. So $e(a) \theta a$ for any $a \in A$. Hence $\mathfrak{A} j^{\prime} \theta \in \Upsilon_{1}$. Since $a, b \in[c] \theta$ implies $e(a)=$ $e(b)=e(c)$, every $\theta$-block belongs to $\tau_{0}$. Thus $\mathscr{H} \in \tau_{0} \cdot \tau \cdot \tau_{1} ;$ i.e., $\Upsilon^{\top}=\tau_{0} \cdot \tau_{\top} \tau_{1}$.

Thus a variety with a unit term satisfying any of the conditions of Theorem 3.8 is the product of a variety whose systems have precisely one unit and an idempotent variety; i.e., a variety in which every element is a unit.

The following statements are corollaries of Theorem 3.8:

A semigroup variety satisfying $x=x^{n+1}$, for a given positive $n$, is the product of some subvariety of groups and some subvariety of bands iff it satisfies $(x y)^{n}=$ $\left(x^{n} y^{n}\right)^{n}$. A weakly congruence regular variety with unit terms is the product of a subvariety with ideals and an idempotent congruence regular subvariety iff it satisfies (i) of Theorem 3.8. (This follows from Theorems 3.7 and 3.8, since every weakly congruence regular idempotent variety is congruence regular [12], and weakly congruence regular varieties with constant terms and unit terms are varieties with ideals.)

4. In this section we study weakly congruence permutable varieties and the syntax of $\cdot$ in .

DEFINITION 4.1. A variety it of systems is weakly congruence permutable if for every $\mathfrak{H}$-system $\mathfrak{A}$, there is a unit $e \in A$ such that for any pair of congruences $\theta, \Phi$ on $\mathcal{H},[[e] \theta] \Phi \subseteq[[e] \Phi] \theta$.

Similar to Mal'cev's Characterization Theorem of congruence permutable varieties, we can prove

THEOREM 4.1. A variety ' ' of systems is weakly congruence permutable iff there exist a unary term $e\left(x_{0}\right)$ and a ternary term $u\left(x_{0}, x_{1}, x_{2}\right)$ in the language of the type of $\tau$ such that $e\left(x_{0}\right)$ is a unit and $u\left(e\left(x_{0}\right), x_{1}, x_{1}\right)=e\left(x_{0}\right)$ and $u\left(e\left(x_{0}\right), e\left(x_{0}\right), x_{1}\right)=x_{1}$. Moreover, if the latter is true, then for every $a \in \mathfrak{H} \in \mathcal{T}$ and every pair of congruences $\theta, \Phi$ on $\mathfrak{A},[[e(a)] \theta] \Phi=[[e(a)] \Phi] \theta$.

Proof. Let is be the 'T-free system on $\omega$ free generators. Let $\tau$ ' be weakly congruence permutable. There is a unit $f=f\left(x_{0}, \ldots, x_{n-1}\right) \in F$ such that for any pair of congruences $\theta, \Phi$ on $\tilde{i},[[f] \theta] \Phi \subseteq[[f] \Phi] \theta$. Let $\theta=\theta\left(f, x_{n}\right), \Phi=$ $\theta\left(x_{n}, x_{n+1}\right)$. Hence $\left(f, x_{n+1}\right) \in \theta \Phi$; i.e., $x_{n+1} \in[[f] \theta] \Phi$. Hence $x_{n+1} \in[[f] \Phi] \theta$; i.e., $\left(f, x_{n+1}\right) \in \Phi \theta$. There is $t=t\left(x_{0}, \ldots, x_{n}, x_{n+1}, \ldots, x_{m}\right) \in F$ such that $(f, t) \in$ $\Phi=\theta\left(x_{n}, x_{n+1}\right)$ and $\left(t, x_{n+1}\right) \in \theta=\theta\left(f, x_{n}\right)$. The endomorphisms of $\mathfrak{F}$ defined by $x_{i} \alpha=x_{i}$ if $i \neq n$ and $x_{n} \alpha=f, x_{i} \beta=x_{i}$ if $i \neq n+1$ and $x_{n+1} \beta=x_{n}$ satisfy

$$
\begin{gathered}
t \alpha=t\left(x_{0}, \ldots, x_{n-1}, f, x_{n+1}, \ldots, x_{m}\right)=x_{n+1} \alpha=x_{n+1}, \\
t \beta=t\left(x_{0}, \ldots, x_{n}, x_{n}, \ldots, x_{m}\right)=f \beta=f .
\end{gathered}
$$


If $\gamma$ is the endomorphism of $\mathfrak{F}$ defined by $x_{i} \gamma=x_{0}$ if $i \neq n$ and $i \neq n+1, x_{n} \gamma=x_{1}$ and $x_{n+1} \gamma=x_{2}, u\left(x_{0}, x_{1}, x_{2}\right)=t \gamma$ and $e\left(x_{0}\right)=f \gamma$ satisfy the condition in the theorem.

Conversely, let $e$ and $u$ satisfy the condition in the theorem. If $a \in \mathfrak{A} \in \mathcal{T}, \boldsymbol{\theta}, \Phi$ are congruences on $\mathfrak{A}$ and $b \in[[e(a)] \theta] \Phi$, then there is $c \in A$ such that $e(a) \theta c$ and $c \Phi b$. Hence $u(e(a), b, b) \Phi u(e(a), c, b)$ and $u(e(a), c, b) \theta u(e(a), e(a), b)$; i.e., $e(a) \Phi u(e(a), c, b)$ and $u(e(a), c, b) \theta b$. Thus $b \in[[e(a)] \Phi] \theta$.

As an example of a weakly congruence permutable variety that is not permutable, consider the groupoid variety defined by: $x x=y y$ and $(x x) y=y$. Here $e(x)=x x$ and $u(x, y, z)=y z$. The groupoid of four elements $\{e, a, b, c\}$, defined by $e x=x$ for all $x$ and $y z=e$ for all $z$ and for all $y \neq e$, belongs to this variety. However,

$$
[[a] \theta(a, b)] \theta(b, c)=\{a, b, c\} \text { and }[[a] \theta(b, c)] \theta(a, b)=\{a, b\} .
$$

Lemma 4.2. Suppose $\mathcal{T}$ is a weakly congruence permutable variety of systems. If $\mathfrak{U} \in \mathcal{T}$ and $a \in A$ is a unit, then for any pair of congruences $\theta, \Phi$ on $\mathfrak{U}$,

$$
[a](\theta \vee \Phi)=[[a] \theta] \Phi=[[a] \Phi] \boldsymbol{\theta} .
$$

Proof. Since $a$ is a unit, $e(a)=a$. Thus by Theorem 4.1, $[[a] \theta] \Phi=[[a] \Phi] \theta$. Let $b \in[a](\theta \vee \Phi)$. Then there are $a_{1}, \ldots, a_{n} \in A$ such that $a_{i} \theta a_{i+1}$ if $i$ is even and $a_{i} \Phi a_{i+1}$ if $i$ is odd and $a \theta a_{1}$ and $a_{n}=b$. Thus $a_{2} \in[[a] \theta] \Phi$ and so $a_{2} \in[[a] \Phi] \theta$. But $a_{2} \theta a_{3}$. Hence $a_{3} \in[[a] \Phi] \theta=[[a] \theta] \Phi$. Thus we can reduce the sequence $a_{1}, \ldots, a_{n}$ to $a_{1}, a_{4}, \ldots, a_{n}$ since $a_{3} \Phi a_{4}$. Thus $a_{4} \in[[a] \theta] \Phi, a \theta a_{1}^{\prime}$ and $a_{1}^{\prime} \Phi a_{4}$. So we can reduce the sequence till we reach a sequence $c_{1}, c_{2}$ where $a \theta c_{1}$ and $c_{1} \Phi c_{2}, c_{2}=b$; i.e., $b \in[[a] \theta] \Phi$; i.e., $[a](\theta \vee \Phi) \subseteq[[a] \theta] \Phi$.

THEOREM 4.3. Suppose $\mathfrak{K}$ is a weakly congruence permutable variety of systems and Q, $\mathcal{T}$ are subvarieties of $\mathcal{K}$. Then $\mathscr{Q} \circ \mathcal{T}$ is closed under homomorphic images and थ. $\cdot \mathcal{T}$ is a subvariety of $\mathcal{K}$.

Proof. Let $\mathfrak{A} \in \mathcal{K}$ and let $\theta$ be a congruence on $\mathfrak{U}$ such that $\mathfrak{A} / \boldsymbol{\theta} \in \mathfrak{T}$. Since there is a unit term $e(x)$, we have for each $a \in A$ that $[a] \theta$ is a subsystem of $\mathscr{U}$ iff there is a unit $e \in[a] \theta$.

Let $[e] \theta \in \mathcal{Q}$, where $e$ is a unit. Let $\alpha$ be a homomorphism of $\mathfrak{A}$ onto $\mathfrak{B} \in \mathfrak{H}$ and let $\Phi$ be the kernel congruence of $\alpha$. Then $\theta \vee \Phi$ is a congruence on $\mathfrak{A}$ and $\theta^{\prime}=(\theta \vee \Phi) \alpha$ is a congruence on $\mathfrak{B}$ such that $\mathfrak{B} / \theta^{\prime}$ is a homomorphic image of $\mathfrak{A} / \boldsymbol{\theta}$. Thus $\mathfrak{B} / \boldsymbol{\theta}^{\prime} \in \mathcal{T}$.

$$
[e \alpha] \theta^{\prime}=([e](\theta \vee \Phi)) \alpha=([[e] \theta] \Phi) \alpha=([e] \theta) \alpha
$$

by Lemma 4.2. So $[e \alpha] \theta^{\prime}$ is a homomorphic image of $[e] \theta \in \mathcal{Q}$. Hence $[e \alpha] \theta^{\prime} \in \mathcal{Q l}$.

Thus if $\mathfrak{A} \in \mathscr{Q} \circ \mathscr{K} \mathcal{T}$, then $\mathfrak{B} \in \mathscr{Q} \circ \mathscr{K}$ r since if $\mathfrak{C}$ is a subsystem of $\mathfrak{A}$ and $\theta$ is a congruence on $\mathfrak{A}$ such that $C$ is a union of $\theta$-blocks, $\mathfrak{} \in \mathscr{U}$ and $\mathfrak{A} / \theta \in \widetilde{\top}, C$ contains a unit $e,[e] \theta$ is a subsystem of $\mathfrak{E}$, so $[e] \theta \in \mathcal{U}$. If $\mathfrak{A} \in \mathcal{Q l} \cdot \mathcal{T} \widetilde{T}$, then $[e] \theta \in \mathcal{Q}$ for every unit $e \in A$, where $\theta$ is a $\mathcal{T}$-congruence on $\mathfrak{A}$. Let $f \in B$ be a unit and $a \alpha=f$ (since $\alpha$ is onto). Hence $e(f)=e(a \alpha)=e(a) \alpha$. Thus there is a unit $e \in A$ such that $e \alpha=f$. But then (as above) $[f] \theta^{\prime}=[e \alpha] \theta^{\prime}=([e] \theta) \alpha$; i.e., $[f] \theta^{\prime} \in$

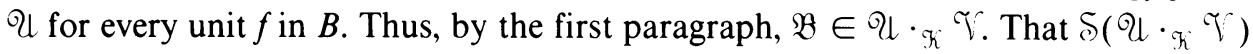
$\subseteq \mathcal{Q} \cdot \Re \mathcal{T}$ and $\mathscr{P}(\mathcal{Q} \cdot \Re \mathcal{T}) \subseteq \mathcal{Q} \cdot \mathcal{T}$ was shown by A. I. Mal'cev in [23]. That 
$\mathcal{Q} \cdot \mathfrak{T}$ is a variety when $\mathscr{K}, \mathcal{Q}, \widetilde{T}$ are congruence permutable varieties was established in [23].

THEOREM 4.4. Suppose $\mathfrak{T}$ is a variety of systems with constant terms. Then $\mathfrak{T}$ is weakly congruence permutable iff there is a binary term $t\left(x_{0}, x_{1}\right)$ such that $t\left(x_{0}, x_{0}\right)=0$ is $a$ unit and $t\left(0, x_{1}\right)=x_{1}$.

Proof. By Lemma 3.6, $\mathcal{T}$ is a variety in which every system has a unique 0 such that 0 is the value taken by every constant term and by every unit term. Theorem 4.4 then follows from Theorem 4.1, where $t\left(x_{0}, x_{1}\right)=u\left(0, x_{0}, x_{1}\right)$ and $e\left(x_{0}\right)=0$.

THEOREM 4.5. If $\mathfrak{T}$ is a weakly congruence permutable and weakly congruence regular variety of systems, then $\mathcal{V}$ is congruence Arguesian (and so congruence modular ). Moreover $\mathcal{T}$ is congruence $n$-permutable for some $n$.

The proof that $\mathcal{T}$ is congruence Arguesian is similar to B. Jónsson's proof [18] that congruence permutability implies congruence Arguesianness.

Proof. Let $\mathfrak{A} \in \mathcal{V}$ and let $e$ be a unit of $\mathfrak{A}$. Since $\mathscr{V}$ has a unit term and is weakly congruence regular, congruences are determined by any block containing a unit, by Proposition 3.3. So, if $\theta, \theta^{\prime}$ are congruences on $\mathfrak{A}$, then $\theta \leqslant \theta^{\prime}$ iff $[e] \theta \subseteq[e] \theta^{\prime}$. Let $\theta_{i}$, $\Phi_{i}, 0 \leqslant i \leqslant 2$, be congruences on $\mathfrak{A}$,

$$
\begin{gathered}
\theta=\cap\left\{\theta_{i} \vee \Phi_{i}: 0 \leqslant i \leqslant 2\right\}, \\
\Psi_{0}=\left[\left(\theta_{0} \vee \theta_{2}\right) \cap\left(\Phi_{0} \vee \Phi_{2}\right)\right] \vee\left[\left(\theta_{1} \vee \theta_{2}\right) \cap\left(\Phi_{1} \vee \Phi_{2}\right)\right], \\
\Psi=\Psi_{0} \cap\left(\theta_{0} \vee \theta_{1}\right) \cap\left(\Phi_{0} \vee \Phi_{1}\right)
\end{gathered}
$$

and

$$
\theta^{\prime}=\left[\theta_{0} \cap\left(\theta_{1} \vee \Psi\right)\right] \vee\left[\Phi_{0} \cap\left(\Phi_{1} \vee \Psi\right)\right]
$$

To show that the congruence lattice is Arguesian it suffices to show that $[e] \theta \subseteq[e] \theta^{\prime}$. Let $e \theta a$. Then $e\left(\theta_{i} \vee \Phi_{i}\right) a, 0 \leqslant i \leqslant 2$. Since $\widetilde{V}$ is weakly congruence permutable, by Lemma $4.2, e \theta_{i} \Phi_{i} a, 0 \leqslant i \leqslant 2$. Now proceed as in the usual proof of permutability implies Arguesianness to obtain $e \theta^{\prime} a$.

$\mathfrak{A}$ is congruence $n$-permutable if for any congruences $\theta, \Phi$ on $\mathfrak{A}, \theta \cdot \Phi \cdot \theta \cdot \Phi$ $\cdots=\Phi \cdot \theta \cdot \Phi \cdot \theta \cdots$, where both sides of the equality are of length $n$ (see $[12,13$, 27, 29, 31, 32, 37]).

Let $\mathfrak{A} \in \mathfrak{V}$, let $e$ be a unit of $\mathfrak{A}$, and let $\theta, \Phi$ be congruences on $\mathfrak{A}$. By Definition 4.1 and Theorem 3.4 $\mathcal{T}$ admits extensions. Let $t_{i}$ and $p_{i j}$ be the terms of condition (iii) of Theorem 3.5. Let $a(\theta \vee \Phi) b$. Then $e(\theta \vee \Phi) t_{i}, t_{i}=t_{i}(e, a, b), 1 \leqslant i \leqslant k$. By Lemma 4.2, $e \theta \Phi t_{i}$ and $e \Phi \theta t_{i}, 1 \leqslant i \leqslant k$. There are $c_{i}, d_{i}$ such that $e \theta c_{i} \Phi t_{i}$ and $e \Phi d_{i} \theta t_{i}, 1 \leqslant i \leqslant k$. Hence

$$
p_{i j}(e, a, b, e) \theta p_{i j}\left(e, a, b, c_{i}\right) \Phi p_{i j}\left(e, a, b, t_{i}\right)
$$

i.e.,

$$
p_{i j}(e, a, b, e) \theta \Phi p_{i j}\left(e, a, b, t_{i}\right)
$$

and, similarly,

$$
p_{i j}(e, a, b, e) \Phi \theta p_{i j}\left(e, a, b, t_{i}\right)
$$


Hence

$$
\begin{gathered}
p_{i 0}(e, a, b, e) \theta \Phi p_{i 0}\left(e, a, b, t_{i}\right)=p_{i 1}\left(e, a, b, t_{i}\right) \\
p_{i 1}(e, a, b, e) \theta \Phi p_{i 1}\left(e, a, b, t_{i}\right) .
\end{gathered}
$$

Hence

$$
p_{i 0}(e, a, b, e) \theta \Phi \theta p_{i 1}(e, a, b, e)=p_{i 2}(e, a, b, e)
$$

Hence

$$
p_{i 0}(e, a, b, e)(\theta \Phi)^{m(i)} p_{i m(i)}\left(e, a, b, t_{i}\right)=p_{(i+1) 0}(e, a, b, e) .
$$

Hence

$$
\begin{aligned}
a=p_{10}(e, & a, b, e)(\theta \Phi)^{m(1)} p_{20}(e, a, b, e)(\theta \Phi)^{m(2)} \\
& \cdots p_{(k-1) 0}(e, a, b, e)(\theta \Phi)^{m(k)} p_{k m(k)}\left(e, a, b, t_{k}\right)=b .
\end{aligned}
$$

Hence $a(\theta \Phi)^{s} b$ where $s=m(1)+\cdots+m(k)$. Thus $\mathscr{V}$ is congruence $2 s$-permutable.

From [3] it follows that congruence regular varieties are congruence modular.

Now we study the syntax of weakly congruence permutable varieties.

LEMMA 4.6. Suppose $\mathfrak{K}$ is a weakly congruence permutable variety of algebras, $\mathfrak{A} \in \mathscr{K}, \mathcal{T}$ is a subvariety of $\mathcal{K}$, and $\theta$ is the $\mathcal{V}$-congruence on $\mathfrak{A}$. Let $\mathfrak{F}$ be the $\mathcal{K}$-free algebra on $\omega$ free generators and let $\alpha$ be a homomorphism of $\mathfrak{F}$ onto $\mathfrak{A}$. If $a \in A$ is a unit, $f=f\left(x_{0}, \ldots, x_{n-1}\right)$ is $a$ unit of $\mathfrak{F}$ and $x_{k} \alpha=a, 0 \leqslant k<n$, then

$$
[a] \theta=\left\{u\left(a, x_{n} \alpha, \ldots, x_{m} \alpha\right) \mid \mathcal{T} \vDash u\left(f, x_{n}, \ldots, x_{m}\right)=f\right\} .
$$

Proof. As $\mathfrak{A} / \boldsymbol{\theta} \in \mathfrak{T}, \mathfrak{A} / \boldsymbol{\theta}$ is a homomorphic image of $\mathfrak{F} / \boldsymbol{\theta}^{\prime}$ where $\boldsymbol{\theta}^{\prime}$ is the $\checkmark$-congruence on $\mathfrak{F}$. Hence $\theta \alpha^{-1} \supseteq \theta^{\prime} \vee$ kernel $\alpha$. Thus $\theta \alpha^{-1}=\theta^{\prime} \vee \Phi$, where $\Phi=$ kernel $\alpha$, since $\mathfrak{F} /\left(\theta^{\prime} \vee \Phi\right) \in \mathcal{V}$ and $\mathfrak{F} /\left(\theta^{\prime} \vee \Phi\right)$ is a homomorphic image of $\mathfrak{A}$ and, therefore, of $\mathfrak{U} / \theta$. Let $c \in[a] \theta$; i.e., $a \theta c$. There is $z \in F$ such that $z \alpha=c$. As $f \alpha=f(a, \ldots, a)=a,(f, z) \in \theta \alpha^{-1}=\theta^{\prime} \vee \Phi$. Hence $z \in[f]\left(\theta^{\prime} \vee \Phi\right)=\left[[f] \theta^{\prime}\right] \Phi$, by Lemma 4.2 , since $\mathcal{K}$ is weakly congruence permutable and $f$ is a unit. Hence there is $t \in F$ such that $f \theta^{\prime} t$ and $t \Phi z$. Thus $t \alpha=z \alpha=c$ and $f=t$ is an identity in $\mathcal{T}$. $t=t\left(x_{0}, \ldots, x_{n-1}, x_{n}, \ldots, x_{m}\right)=f$ is an identity in $\mathcal{T}$. So $t\left(f, \ldots, f, x_{n}, \ldots, x_{m}\right)=$ $f(f, \ldots, f)=f$ is an identity in $\mathcal{T}$. Let $u\left(x_{0}, x_{n}, \ldots, x_{m}\right)=t\left(x_{0}, \ldots, x_{0}, x_{n}, \ldots, x_{m}\right)$. Then $u\left(f, x_{n}, \ldots, x_{m}\right)=f$ is an identity in $\mathcal{V}$ and

$$
c=t \alpha=t\left(a, \ldots, a, x_{n} \alpha, \ldots, x_{m} \alpha\right)=u\left(a, x_{n} \alpha, \ldots, x_{m} \alpha\right) \text {. }
$$

Hence

$$
[a] \theta \subseteq\left\{u\left(a, x_{n} \alpha, \ldots, x_{m} \alpha\right) \mid \mathcal{T}_{F} u\left(f, x_{n}, \ldots, x_{m}\right)=f\right\} .
$$

The inverse inclusion is obvious.

LEMMA 4.7. Suppose $\mathcal{K}$ is a weakly congruence permutable variety of algebras and $\mathfrak{i}$ is the $\mathcal{K}$-free algebra on $\omega$ free generators. If $f_{i}=f_{i}\left(x_{0}, \ldots, x_{n-1}\right)$ is a unit of $\mathcal{F}$, $i=1,2$, and $\theta$ is a fully invariant congruence on $\mathfrak{F}$, then

$$
\left[f_{1}\right] \theta=\left\{u\left(f_{1}, x_{0}, \ldots, x_{m}\right) \mid u\left(f_{2}, x_{n}, \ldots, x_{n+m}\right) \in\left[f_{2}\right] \theta\right\} .
$$


Proof. Let $\alpha$ be the endomorphism into is defined by $x_{k} \alpha=f_{1}$ if $k<n$ and $x_{k} \alpha=x_{k-n}$ if $k \geqslant n$. Then apply Lemma 4.6 where $\mathfrak{U}=\mathfrak{r}, a=f_{1}, f=f_{2}$ and $\dddot{\Upsilon}$ is the variety generated by $i / \theta$.

LEMma 4.8. Suppose ith is a weakly congruence permutable variety of algebras, is is the th-free algebra on $\omega$ free generators and $\theta, \Phi$ are fully invariant congruences on $\mathfrak{F}$. Suppose $f_{i}=f_{i}\left(x_{0}, \ldots, x_{n-1}\right), u_{i}\left(x_{1}, \ldots, x_{m}\right), i=1,2$, are terms such that $f_{1}, f_{2}$ are units of is and $\left(u_{1}\left(v_{1}, \ldots, v_{m}\right), u_{2}\left(v_{1}, \ldots, v_{m}\right)\right) \in \Phi$ for all $v_{1}, \ldots, v_{m} \in\left[f_{1}\right] \theta$. Then $\left(u_{1}\left(t_{1}, \ldots, t_{m}\right), u_{2}\left(t_{1}, \ldots, t_{m}\right)\right) \in \Phi$ for all $t_{1}, \ldots, t_{m} \in\left[f_{2}\right] \theta$.

Proof. Let $\alpha$ be the endomorphism onto is defined by $x_{k} \alpha=f_{2}$ if $k<n$ and $x_{k} \alpha=x_{k-n}$ if $k \geqslant n$. By Lemma 4.7, $\left[f_{2}\right] \theta \subseteq\left(\left[f_{1}\right] \theta\right) \alpha$. Let $t_{1}, \ldots, t_{m} \in\left[f_{2}\right] \theta$. Then $t_{i}=v_{i} \alpha, 1 \leqslant i \leqslant m$, where $v_{1}, \ldots, v_{m} \in\left[f_{1}\right] \theta$. Then $u_{i}\left(t_{1}, \ldots, t_{m}\right)=u_{i}\left(v_{1}, \ldots, v_{m}\right) \alpha$, $i=1,2$. Since $\left(u_{1}\left(v_{1}, \ldots, v_{m}\right), u_{2}\left(v_{1}, \ldots, v_{m}\right)\right) \in \Phi, \Phi$ is fully invariant and $\alpha$ is an endomorphism, $\left(u_{1}\left(v_{1}, \ldots, v_{m}\right) \alpha, u_{2}\left(v_{1}, \ldots, v_{m}\right) \alpha\right) \in \Phi$.

LEMMA 4.9. Suppose $\mathrm{th}$ is a weakly congruence permutable variety of algebras, $\mathcal{Q}, \tau$,

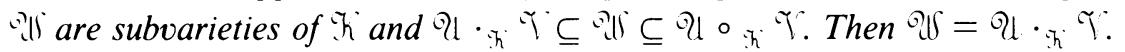

Proof. Let is be the free $h$-algebra on $\omega$ free generators. Using Theorem 4.3,

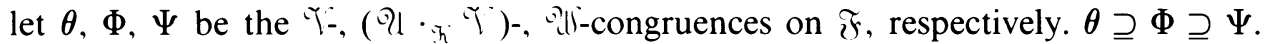

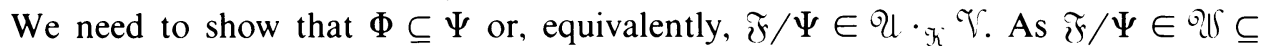
Q1 $\circ \mathrm{T}$, by Definition 5 , there is a subalgebra $\mathscr{B}$ of $\mathfrak{F} / \Psi$ that is a union of blocks of the $\mathcal{T}$-congruence on $\tilde{F} / \Psi$ and which satisfies $\mathcal{B} \in \mathcal{Q}$. Thus there is a subalgebra $\sqrt{5}$ of $\mathfrak{F}$ that is a union of $\theta$-blocks and $\widetilde{c} /\left(C^{2} \cap \Psi\right) \in \mathcal{Q}$. Since there are unit terms on $\mathcal{T}$, let $e$ be a unit of $\mathfrak{C}$. Hence $[e] \theta /\left(([e] \theta)^{2} \cap \Psi\right) \in \mathcal{Q}$, as it is isomorphic to a subalgebra of $\mathfrak{s} /\left(C^{2} \cap \Psi\right) \in \mathcal{Q}$. Let $f$ be a unit of $\tilde{f}$ and let $u_{1}=u_{2}$ be an identity in 21 . Then $\left(u_{1}\left(v_{1}, \ldots, v_{m}\right), u_{2}\left(v_{1}, \ldots, v_{m}\right)\right) \in \Psi$ for all $v_{1}, \ldots$, $v_{m} \in[e] \theta$. By Lemma 4.8, $\left(u_{1}\left(t_{1}, \ldots, t_{m}\right), u_{2}\left(t_{1}, \ldots, t_{m}\right)\right) \in \Psi$ for all $t_{1}, \ldots, t_{m} \in$ $[f] \theta$; i.e., $[f] \theta /\left(([f] \theta)^{2} \cap \Psi\right) \in \mathcal{Q l}$. Thus every block of $\theta / \Psi$ that is a subalgebra of $\tilde{T} / \Psi$ is in $\vartheta$; i.e., $\bar{T} / \Psi \in \mathcal{Q} \cdot \pi$.

COROLlARY 4.10. Suppose th is a weakly congruence permutable variety of algebras,

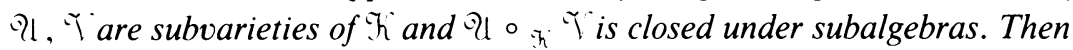

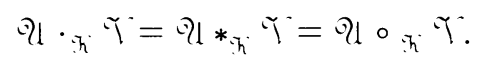

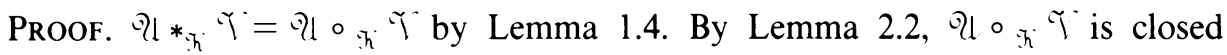
under Cartesian products, and by Theorem 4.3, it is closed under homomorphic images. Thus $2 \circ^{\circ} T$ is a subvariety of $\mathfrak{K}$, and by Theorem 4.1 and Definition 5 Q1 $\cdot$; $T \subseteq Q 1 \circ ; \%$. The corollary follows from Lemma 4.9.

THEOREM 4.11. Suppose $\mathfrak{h}$ is a weakly congruence permutable variety of algebras, Q1, Tare subvarieties of $\mathrm{T}, \mathrm{f}$ is a unit term on $\mathrm{T}$ and $\Sigma$ is a basis of identities for $\mathrm{Ql}$. Then $\mathrm{Q}_{1} \cdot \mathrm{T}$ is defined relative to th by the identities:

$$
\left\{u_{1}\left(v_{1}, \ldots, v_{m}\right)=u_{2}\left(v_{1}, \ldots, v_{m}\right) \mid u_{1}=u_{2} \in \Sigma, T=v_{i}=f, 1 \leqslant i \leqslant m\right\} .
$$

Proof. Let is be the $t h$-free algebra on $\omega$ free generators. We can assume $f=f\left(x_{0}, \ldots, x_{n-1}\right) \in F$. Let $\theta, \Phi$ be the $T-,\left(\vartheta \cdot \tau_{i} T\right)$-congruences on $\mathcal{F}$, respectively. It is clear that $\widetilde{F} / \Phi$ satisfies the identities $u_{1}\left(v_{1}, \ldots, v_{m}\right)=u_{2}\left(v_{1}, \ldots, v_{m}\right)$ for 
all $u_{1}=u_{2} \in \Sigma$ and $v_{1}, \ldots, v_{m}$ such that $v_{i}=f$ is an identity in $\mathcal{T}, 1 \leqslant i \leqslant m$. Let 915 be the subvariety of $\mathscr{K}$ defined by these identities. Hence $\mathscr{U} \cdot \mathcal{T} \subseteq \vartheta_{l l}$. By Lemma 4.9 , it suffices to show that $\mathscr{Q} \subseteq \mathcal{Q} \circ \% \mathcal{T}$. Let $\Psi$ be the $\mathcal{Q}$-congruence on $\mathfrak{F}$. Hence for all $v_{1}, \ldots, v_{m} \in[f] \theta, u_{1}=u_{2} \in \Sigma$ we have $\left(u_{1}\left(v_{1}, \ldots, v_{m}\right), u_{2}\left(v_{1}, \ldots, v_{m}\right)\right) \in \Psi$. Thus $[f] \theta /\left(([f] \theta)^{2} \cap \Psi\right) \in \mathcal{Q l}_{\text {; }}$ i.e., $\mathfrak{F} / \Psi \in \mathcal{Q}_{l} \circ: \mathfrak{T}$.

COROLlaRy 4.12. Suppose $\mathfrak{K}$ is a weakly congruence permutable variety of algebras

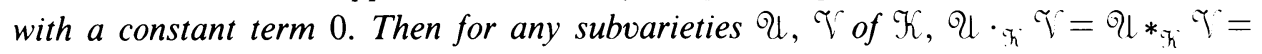
Q1 $\circ$. T T is defined relative to $\mathcal{K}$ by the identities:

$$
\left\{u_{1}\left(v_{1}, \ldots, v_{m}\right)=u_{2}\left(v_{1}, \ldots, v_{m}\right) \mid u_{1}=u_{2} \in \Sigma, \mathcal{T} \vDash v_{i}=0,1 \leqslant i \leqslant m\right\}
$$

where $\Sigma$ is a basis of identities for 91.

Corollary 4.12 follows from Lemmas 2.1 and 3.6 and Corollary 4.10 and Theorem 4.11.

COROLlARY 4.13. Suppose $\mathfrak{H}$ is a variety of algebras that is both weakly congruence regular and weakly congruence permutable, $\mathcal{Q}, \mathcal{T}$ are subvarieties of $\mathcal{K}$ and $\Sigma$ is a basis of identities of $\mathcal{Q}$ of the form $u\left(f, x_{n}, \ldots, x_{s}\right)=f$, where $f$ is a unit of $\mathfrak{F}$, the free Th-algebra on $\omega$ free generators. Then $\mathscr{Q l}_{\mathrm{T}} \mathrm{T}$ T is defined relative to $\mathscr{K}$ by the identities:

$$
\begin{aligned}
\left\{u\left(f, v_{0}, \ldots, v_{k}\right)=f \mid u(\right. & \left.f, x_{n}, \ldots, x_{n+k}\right)=f \in \Sigma, \\
& \left.\Upsilon \vDash v_{i}\left(f, x_{n}, \ldots, x_{n+m}\right)=f, 0 \leqslant i \leqslant k\right\} .
\end{aligned}
$$

$\mathscr{Q}$ always has such a basis, in part because, by Proposition 3.3, every congruence of $\mathfrak{F}$ is determined by its $f$-block.

Corollary 4.13 gives the syntax of $\mathscr{U} \cdot \mathcal{T}$ where $\mathcal{K}$ is the variety of idempotent quasigroups, loops, groups, rings, modules, etc.

An abstract characterization of idempotent medial quasigroups is in [8].

COROLlaRY 4.14. Suppose $\mathfrak{K}$ is a variety of algebras with a constant term 0 that is both weakly congruence regular and weakly congruence permutable, $\mathcal{Q}, \mathcal{T}$ are subvarieties of $\mathcal{K}, \mathfrak{F}$ is the free $\mathcal{K}$-algebra on $\omega$ free generators and $U, V$ are the 0 -blocks of

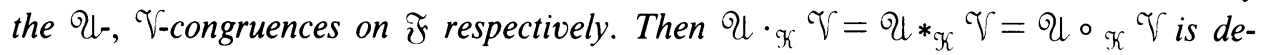
fined relative to $\mathcal{K}$ by $u\left(v_{0}, \ldots, v_{m}\right)=0$, where $u\left(x_{0}, \ldots, x_{m}\right) \in U$ and $v_{i} \in V, 0 \leqslant i$ $\leqslant m$.

Corollary 4.14 follows from Lemmas 2.1 and 3.6 and Corollaries 4.10 and 4.13.

Corollary 4.14 gives the form of identities of $\mathscr{U} \cdot \mathcal{K}=\mathcal{Q}_{*_{K}} \mathfrak{V}=\mathscr{Q} \circ \mathcal{K}$ using the fully invariant subgroups when $\mathcal{K}$ is a group variety [28], and $T$-ideals when $\mathscr{K}$ is a ring variety [15]. This also gives the form of identities for the case of loops, multioperator groups, etc. 


\section{REFERENCES}

1. S. L. Bell and A. B. Slomson, Models and ultraproducts: an introduction, North-Holland, Amsterdam, 1969.

2. G. Birkhoff, Lattice theory, 3rd ed., Amer. Math. Soc. Colloq. Publ., vol. 25, Amer. Math. Soc., Providence, R. I., 1967.

3. S. Bullman-Fleming, A. Day and W. Taylor, Regularity and modularity of congruences, Algebra Universalis 4 (1974), 58-60.

4. A. Clifford, Extensions of semigroups, Trans. Amer. Math. Soc. 68 (1950), 165-173.

5. P. M. Cohn, Universal algebra, Harper \& Row, New York, 1965; Rev. ed., Reidel, Dordrecht and Boston, 1981.

6. B. Csákány, Characterization of regular varieties, Acta Sci. Math. (Szeged) 31 (1970), 187-189.

7. __ Congruences and subalgebras, Ann. Univ. Sci. Budapest Eötvös Sect. Math. 18 (1975). 37-44.

8. B. Csákány and L. Megyesi, Varieties of idempotent medial quasigroups, Acta Sci. Math. (Szeged) 37 (1975), 17-23.

9. C. J. Everett, An extension theory for rings, Amer. J. Math. 64 (1942), 363-370.

10. K. Fichtner, Varieties of universal algebras with ideals, Mat. Sb. 75 (117) (1968), 445-453 = Math. USSR Sbornik 4 (1968), 411-418.

11. G. Grätzer, Universal algebra, Van Nostrand, Princeton, 1968; 2nd ed., Springer-Verlag, New York and Berlin, 1979.

12. ._. Two Mal'cev-type conditions in universal algebra, J. Combin. Theory 8 (1970), 334-342.

13. J. Hagemann and A. Mitschke, On n-permutable congruences, Algebra Universalis 3 (1973), 8-12.

14. L. Henkin, J. D. Monk and A. Tarski, Cylindric algebras, North-Holland, Amsterdam, 1971.

15. A. A. Iskander, Product of ring varieties and attainability, Trans. Amer. Math. Soc. 193 (1974), $231-238$.

16. Coverings in the lattice of varieties, Contributions to Universal Algebra, Colloq. Math. Soc. János Bolyai, vol. 17, North-Holland, 1975, pp. 189-203.

17. B. Jonsson, On the representation of lattices, Math. Scand. 1 (1953), 193-206.

18. _. Modular lattices and Desargues' theorem, Math. Scand. 2 (1954), 295-314.

19. __ Algebras whose congruence lattices are distributive, Math. Scand. 21 (1967), 110-121.

20. __ Sums of finitely based lattice theories, Adv. in Math. 14 (1974), 454-468.

21. B. Jónsson and E. Nelson, Relatively free products in regular varieties, Algebra Universalis 4 (1974), $14-19$.

22. A. I. Mal'cev, On the general theory of algebraic systems, Mat. Sb. 35(77) (1954), 3-20; English transl., Amer. Math. Soc. Transl. (2) 27 (1963), 125-142.

23. On multiplication of classes of algebraic systems, Sibirsk Math. Z. 8 (1967), 346-365. (Russian)

24. __ Algebraic systems, "Nauka”, Moscow, 1970; English transl., Grundlagen Math. Wissenschaften, Bd. 192, Springer-Verlag, Berlin and New York, 1973.

25. The metamathematics of algebraic systems. Collected papers 1936-1967, translated and edited by B. F. Wells III, North-Holland, Amsterdam, 1971.

26. R. McKenzie, Equational bases and nonmodular lattice varieties, Trans. Amer. Math. Soc. 174 (1972), $1-44$.

27. A. Mitschke, Implication algebras are 3-permutable and 3-distributive, Algebra Universalis 1 (1971), 182-186.

28. H. Neumann, Varieties of groups, Springer-Verlag, New York, 1967.

29. R. W. Quakenbush, Models for Mal'cev conditions: n-permutability, Notices Amer. Math. Soc. 18 (1971), 806. Abstract 71T-A182.

30. L. Redei, Die Verallgemeinerung der Schreierschen Erweiterungstheorie, Acta Sci. Math. (Szeged) 14 (1952), 252-273.

31. E. T. Schmidt, Kongruenzrelationen algebraischer Strukturen, Math. Forschungsberichte 25, VEB Deutscher Verlag Wiss., Berlin, 1969.

32. __ On n-permutable equational classes, Acta Sci. Math. (Szeged) 33 (1972), 29-30.

33. O. Schreier, Uber die Erweiterung von Gruppen. I, II, Monatsh. Math. Phys. 34 (1926), 165-180.

34. J. Slominski, On the determining of the form of congruences in abstract algebras with equationally definable constant elements, Fund. Math. 48 (1960), 325-341. 
35. D. M. Smirnov, Regular varieties of algebras, Algebra i Logika 15 (1976), 331-342 = Algebra and Logic 15 (1977), 207-214.

36. W. Taylor, Characterizing Mal'cev conditions, Algebra Universalis 3 (1973), 351-397.

37. R. Wille, Kongruenzklassengeometrien, Lecture Notes in Math., vol. 113, Springer-Verlag, Berlin, 1970.

38. G. I. Žitomirskiı̄, On extensions of universal algebras, Uspehi Mat. Nauk 29 (1974), 169-170. (Russian)

Department OF MATHEMatics and Statistics, UNiversity OF SOUTHWESTERN Louisiana, LAFAYETTE, LOUISIANA 70504 